\title{
Contribution of the Pulvinar and Lateral Geniculate Nucleus to the Control of Visually Guided Saccades in Blindsight Monkeys
}

\author{
${ }^{\circledR}$ Norihiro Takakuwa, ${ }^{1,2}$ - Kaoru Isa, ${ }^{1,2}$ Hirotaka Onoe, ${ }^{1,3}$ Jun Takahashi, ${ }^{4}$ and ${ }^{-}$Tadashi Isa ${ }^{1,2,3,5}$ \\ ${ }^{1}$ Department of Neuroscience, Graduate School of Medicine, Kyoto University, Kyoto 606-8501, Japan, ${ }^{2}$ Department of Developmental Physiology, \\ National Institute for Physiological Sciences, Okazaki 444-8585, Japan, ${ }^{3}$ Human Brain Research Center, Graduate School of Medicine, Kyoto \\ University, Kyoto 606-8507, Japan, ${ }^{4}$ Department of Clinical Application, Center for iPS Cell Research and Application, Kyoto University, Kyoto 606- \\ 8507, Japan, and ${ }^{5}$ Institute for the Advanced Study of Human Biology (WPI-ASHBi), Kyoto University, Kyoto 606-8501, Japan
}

After damage to the primary visual cortex (V1), conscious vision is impaired. However, some patients can respond to visual stimuli presented in their lesion-affected visual field using residual visual pathways bypassing V1. This phenomenon is called "blindsight." Many studies have tried to identify the brain regions responsible for blindsight, and the pulvinar and/or lateral geniculate nucleus (LGN) are suggested to play key roles as the thalamic relay of visual signals. However, there are critical problems regarding these preceding studies in that subjects with different sized lesions and periods of time after lesioning were investigated; furthermore, the ability of blindsight was assessed with different measures. In this study, we used double dissociation to clarify the roles of the pulvinar and LGN by pharmacological inactivation of each region and investigated the effects in a simple task with visually guided saccades (VGSs) using monkeys with a unilateral V1 lesion, by which nearly all of the contralesional visual field was affected. Inactivating either the ipsilesional pulvinar or LGN impaired VGS toward a visual stimulus in the affected field. In contrast, inactivation of the contralesional pulvinar had no clear effect, but inactivation of the contralesional LGN impaired VGS to the intact visual field. These results suggest that the pulvinar and LGN play key roles in performing the simple VGS task after V1 lesioning, and that the visuomotor functions of blindsight monkeys were supported by plastic changes in the visual pathway involving the pulvinar, which emerged after V1 lesioning.

Key words: blindsight; lateral geniculate nucleus; monkey; pulvinar; saccadic eye movement

Significance Statement

Many studies have been devoted to understanding the mechanism of mysterious symptom called "blindsight," in which patients with damage to the primary visual cortex (V1) can respond to visual stimuli despite loss of visual awareness. However, there is still a debate on the thalamic relay of visual signals. In this study, to pin down the issue, we tried double dissociation in the same subjects (hemi-blindsight macaque monkeys) and clarified that the lateral geniculate nucleus (LGN) plays a major role in simple visually guided saccades in the intact state, while both pulvinar and LGN critically contribute after the V1 lesioning, suggesting that plasticity in the visual pathway involving the pulvinar underlies the blindsight.

Received Sep. 1, 2020; revised Nov. 23, 2020; accepted Dec. 9, 2020.

Author contributions: N.T. and T.I. designed research; N.T. performed research; N.T. and K.I. analyzed data; N.T., K.I., H.O., J.T., and T.I. wrote the paper.

This study was supported by Core Research for Evolutional Science and Technology/Japan Science and Technology Agency (Grant JPMJCR1651), Grant-in-Aid for Scientific Research Kiban (S; Grant 26221003) and Kiban (A; Grant 19H01011) from the Japan Society for the Promotion of Science (JSPS), and Grant-in Aid for Scientific Research on Innovative Areas "Hyper-Adaptation" (Grant No. 19H05723) from JSPS to T.I. We thank Morio Togawa, Jiro Yamashita, Masashi Nakamura, Yuta Shinto, and Kei Kubota for technical assistance; Yoshinori Koshimizu for histological assessments; and Chih-Yang Chen for advice on the analysis of eye movement kinematics.

N. Takakuwa's present address: Max Planck Institute for Brain Research, 60438 Frankfurt am Main, Germany.

The authors declare no competing financial interests.

Correspondence should be addressed to Tadashi Isa at isa.tadashi.7u@kyoto-u.ac.jp.

https://doi.org/10.1523/JNEUROSCI.2293-20.2020

Copyright $\odot 2021$ the authors

\section{Introduction}

After damage to the primary visual cortex (V1), visual awareness is impaired; however, some patients can respond to visual stimuli by manual responses or saccadic eye movements without visual awareness, if the choices are forced (Pöppel et al., 1973; Sanders et al., 1974; Weiskrantz et al., 1974). This phenomenon is called "blindsight." Studying the neural mechanisms of blindsight is expected to shed light on the neural basis of the unconscious processes of visual perception and behavioral control.

In terms of the brain regions responsible for blindsight, the observation with extensive ablation of the cortical tissue suggests the totally subcortical processing for some visuomotor functions (Tomaiuolo et al., 1997; Savina and Guitton, 2018); but other 
studies suggest the involvement of cortical visual processing for goal-directed movements in the blindsight subjects (monkeys: Schmid et al., 2010; Bridge et al., 2019; humans: Ajina et al., 2015; Ajina and Bridge, 2018). As for the visual pathway to the cortex, the earlier studies focused on the role of the superior colliculus (SC)-pulvinar-extrastriate pathway to bypass the V1 (Diamond and Hall, 1969; Bender, 1983, 1988; Warner et al., 2015). The role of SC has been repeatedly confirmed both in monkeys (Mohler and Wurtz, 1977; Rodman et al., 1990; Kato et al., 2011) and in human patients (Leh et al., 2010; Georgy et al., 2016). Later anatomic studies have shown that some portion of the pulvinar directly receive the retinal inputs, which could convey the visual signal to the cerebral cortex (Kaas and Lyon, 2007; Gattass et al., 2014). In contrast, the direct route from the lateral geniculate nucleus (LGN) to the extrastriate cortex has been suggested to play a role in blindsight (Cowey and Stoerig, 1989). Schmid et al. (2010) showed that visual responses in the extrastriate visual areas disappeared after inactivation of the LGN during passive viewing after V1 lesioning in a macaque model. After the inactivation, visually guided saccades (VGSs) were also impaired. Robust visual responses in LGN have been observed in common marmosets after the V1 lesion ( $\mathrm{Yu}$ et al., 2018). Moreover, the involvement of LGN has also been suggested by diffusion tensor MR imaging in human patients (Ajina et al., 2015; Ajina and Bridge, 2018). Thus, the thalamic regions that relay visual signals to the cortex to support blindsight are still unclear. More recently, our laboratory showed that VGS was impaired by inactivation of the ipsilesional pulvinar both by injections of muscimol and by selective blockade of the SC-to-pulvinar pathway by double viral vector infection (Kinoshita et al., 2012, 2019).

Both thalamic nuclei can send signals to the extrastriate cortex (Cowey and Stoerig, 1989; Hendry and Yoshioka, 1994; Berman and Wurtz, 2010). However, we cannot directly compare the roles of the LGN and pulvinar with these contradictory studies, because there are several critical differences among the subjects in these studies, such as the size of the lesion, the way the lesion was made, and the time after lesioning. Furthermore, the experimental measures for assessment were also different. Therefore, in this study, to draw a clear conclusion on the roles of the pulvinar and LGN in blindsight, we designed an inactivation study in the same subjects using the same behavioral task.

We used monkeys with a lesion of a major portion of the unilateral V1 and adopted a simple VGS task to assess their ability, because the damage to V1 was extensive and blindsight was defined as the ability to respond behaviorally to visual stimuli without conscious visual experience in the original literature (Sanders et al., 1974; Weiskrantz et al., 1974; Barbur et al., 1993). Here, neural activity in either the pulvinar or LGN on the ipsilesional or contralesional side was inactivated by microinjection of the $\mathrm{GABA}_{\mathrm{A}}$ receptor agonist muscimol. This approach allowed us to clarify the roles of the pulvinar and LGN in processing visual information in the intact and lesionaffected visual hemifields.

\section{Materials and Methods}

Animal preparation. Two adult Japanese monkeys (Macaca fuscata; both female; body weight, 5-7 kg; monkeys $\mathrm{O}$ and $\mathrm{T}$ ) were used in this study. A head post and chambers were attached for fixing their head position and for inserting an injection needle during the experiments, under anesthesia introduced with xylazine hydrochloride $(2 \mathrm{mg} / \mathrm{kg})$ and ketamine hydrochloride $(5 \mathrm{mg} / \mathrm{kg})$ and maintained with isoflurane (1.0$1.5 \%)$. They were trained on the VGS task. All of the experimental
A

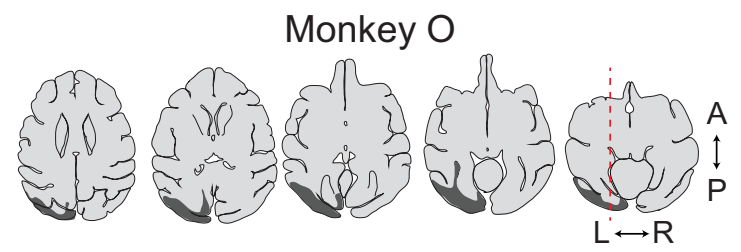

Monkey T

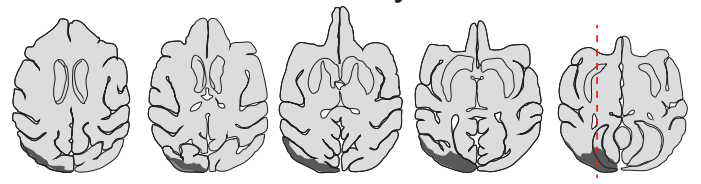

B
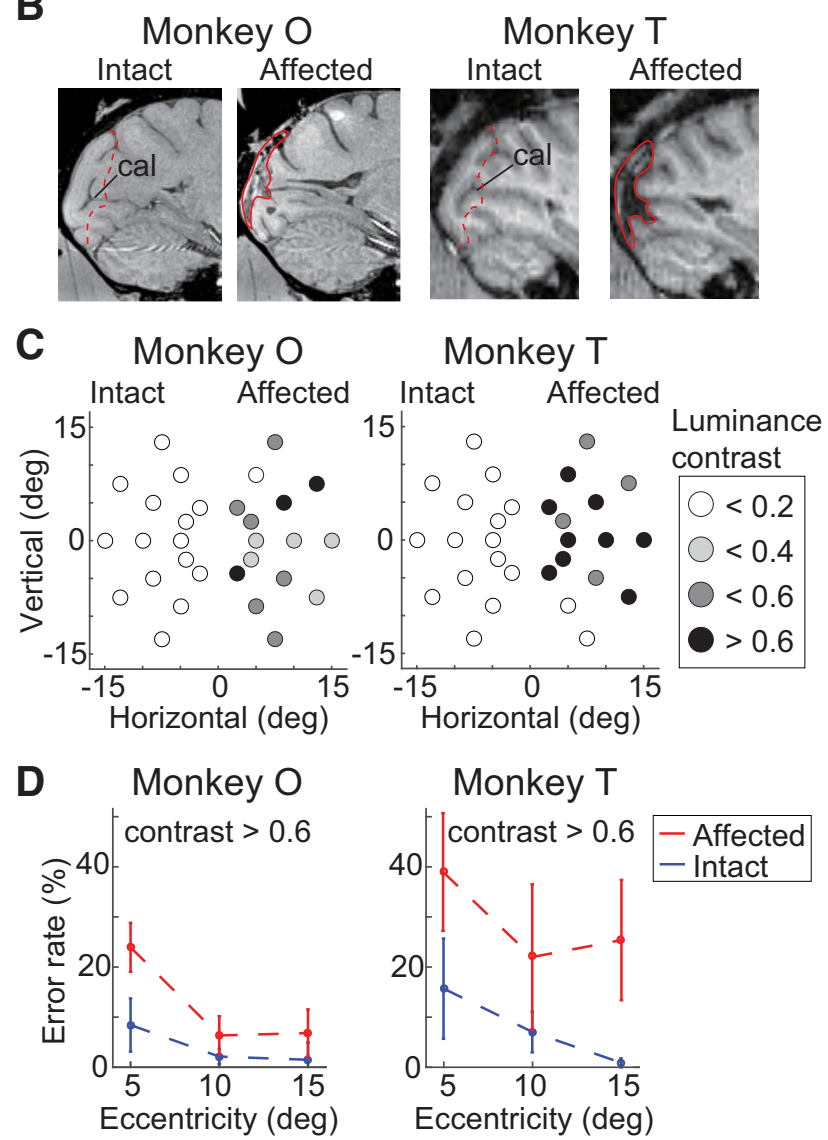

Figure 1. Unilateral V1 lesion and VGS performance. $\boldsymbol{A}$, Lesion areas are indicated in gray in the cartoon coronal brain images. Five horizontal sections of the brains from monkeys 0 and $T$ are presented from left to right from dorsal to ventral $(A$, anterior; $P$, posterior; $L$, left; $R$, right). Red dashed lines in the rightmost panel indicate mediolateral levels of the sagittal sections shown in $\boldsymbol{B}$. $\boldsymbol{B}$, Lesion areas are indicated by red colors on sagittal sections of MR images. Dashed lines show the estimated lesion areas plotted on images of the intact side. The calcarine sulcus (cal) is indicated in the panels of the intact side. C, Deficit maps of each monkey based on the threshold of target luminance contrast by which the monkey could localize the STs. D, Error rate of the VGS task in monkeys 0 and T. In this case, target contrast was $>0.6$. Error rates in the affected visual field and intact visual field are shown in red and blue, respectively.

procedures were performed in accordance with the National Institutes of Health Guidelines for the Care and Use of Laboratory Animals and were approved by the Committees for Animal Experiments at the Graduate School of Medicine in Kyoto University and at the National Institute of Natural Sciences.

Unilateral V1 lesion. Details of the surgical procedures to make the animal model of blindsight have been described previously (Yoshida et al., 2008). Briefly, the left V1 of each monkey was surgically removed by aspiration under the anesthesia described above. As shown in Figure 1, 
$A$ and $B$, the caudal surface of the $\mathrm{V} 1$, except that receiving visual inputs from the foveal region, was removed. Furthermore, caudal aspects of the calcarine sulcus were also aspirated (Fig. $1 B$ ). The lesion also partly included the neighboring V2 and underlying white matter. To assess the extent of the lesion-affected visual field, we used the VGS task and constructed a deficit map.

In this assessment, the luminance contrast of the saccadic target (ST) was changed randomly between 0.02 and 0.95 by random number generator in MATLAB (Weber contrast, 0.04-37.8). Sensitivity to luminance contrast was defined as the minimum contrast at which the monkeys could perform saccadic eye movements with a $>60 \%$ success rate. Deficit maps of individual monkeys were constructed with these values, and the extent of the affected visual field was assessed (Fig. 1C). In general, the visual field disrupted by the V1 lesion extended at least from eccentricities of $5-15^{\circ}$ in both monkeys. Monkey T could perform accurate saccade to STs presented at $300^{\circ}$ in eccentricity of $10^{\circ}$ and $15^{\circ}$ with no change in the sensitivity to luminance contrast, suggesting that the subregion of V1 representing this part of visual field spared the lesion. For these reasons, saccades toward these STs in monkey T were removed from further analysis. The monkeys were used for the present experiments at 1-5 months (monkey O) and 40-47 months (monkey T) after $\mathrm{V} 1$ lesioning, and then killed for histologic assessments.

Reversible inactivation of the pulvinar and LGN. To inactivate neural activity in the pulvinar and LGN, we used microinjection of the $\mathrm{GABA}_{\mathrm{A}}$ receptor agonist muscimol. Muscimol (dissolved in saline; concentration, $1.0 \mu \mathrm{g} / \mu \mathrm{l}$; total injection volume, $0.5-2.0 \mu \mathrm{l})$ was pressure injected $(0.1-$ $0.4 \mu \mathrm{l} / \mathrm{min}$ ) through a 27 gauge needle connected to a $10 \mu \mathrm{l}$ Hamilton syringe. Injection volume and speed were controlled by a syringe pump (Legato 130, Muromachi Kikai). Before starting the muscimol injection experiments, we injected gadolinium (OMNISCAN Intravenous Injection, Daiichi-Sankyo; dissolved in saline at a concentration of $0.02 \mu \mathrm{mol} / \mu \mathrm{l}$; pressure injected at $0.1 \mu \mathrm{l} / \mathrm{min}$; total injection volume, $0.5 \mu \mathrm{l}$ ) into the intended position through a grid in the chamber for fixing the microsyringe with holes at $1 \mathrm{~mm}$ intervals (6-YGD-D1, Crist Instrument), and $1 \mathrm{~h}$ later took magnetic resonance images with a $3 \mathrm{~T}$ MRI scanner (Verio, Siemens) to confirm the injection sites either in the pulvinar or LGN (Fig. 2A,B). This dose of gadolinium can be considered to be nontoxic according to the literature (Alkhunizi et al., 2020). Injection sites of muscimol were later reconstructed according to the coordinates in this MR image of gadolinium and postmortem histologic assessment of injection needle tracks.

In a daily session, the monkeys were required to perform the VGS task for $\sim 20$ min before the muscimol injection as a control (only toward STs with an eccentricity of $10^{\circ}$ ), and we injected muscimol into either the pulvinar or LGN. At 20 min after injection, the VGS task was restarted with ST eccentricity changed in each $10 \mathrm{~min}$ block $\left(10^{\circ}, 5^{\circ}, 15^{\circ}\right.$, and $10^{\circ}$ on the side contralateral to the injection). The control data for STs with other eccentricities $\left(5^{\circ}\right.$ and $\left.15^{\circ}\right)$ were collected on the other experimental days with no muscimol injection.

Eye movement recording system, VGS task design, and statistical analysis. Psychophysics Toolbox version 3 (PTB-3; http://psychtoolbox. org/) on MATLAB (MathWorks) was used for stimulus plotting and data recording. A monitor [Diamondcrysta WIDE RDT272WX (BK), Mitsubishi] was placed at $600 \mathrm{~mm}$ from the eyes of the monkeys. Eye movements were recorded by an eye tracker (EyeLink 1000 PLUS, SR Research) at a sampling rate of $1000 \mathrm{~Hz}$. All statistical analysis in this study was performed using MATLAB software (MathWorks).

In our VGS task, an initial fixation point (FP; size, $0.29^{\circ}$ radius) appeared at the center of the screen. The monkeys had to maintain their gaze in a window ( $1.4^{\circ}$ radius) centered on the FP for 1.0-1.5 s. Another visual stimulus (ST; $0.29^{\circ}$ radius) was then presented randomly at one of five possible locations, each separated by $30^{\circ}$ [centered $\left(0^{\circ}\right)$ on the horizontal meridian], in the hemi-visual field. When the ST appeared, the FP disappeared and the monkeys were required to make a saccade to the ST. The correct window for the ST (eccentricity, $5^{\circ}$; radius, $1.3 \%$ eccentricity, $10^{\circ}$, radius, $2.6^{\circ}$ eccentricity, $15^{\circ}$, radius, $3.9^{\circ}$ ) was a circle with a radius of half the distance between the centers of two neighboring targets [radius $=$ eccentricity $\times \sin$ (direction angle between neighboring target positions)/2]. This arrangement prevented the targets overlapping with
A
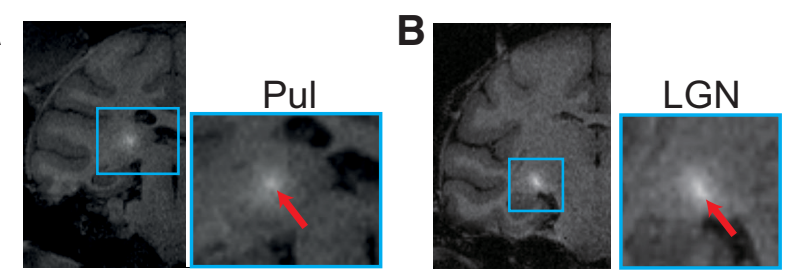

C

O Monkey O

$\bigcirc$ Monkey $\mathrm{T}$
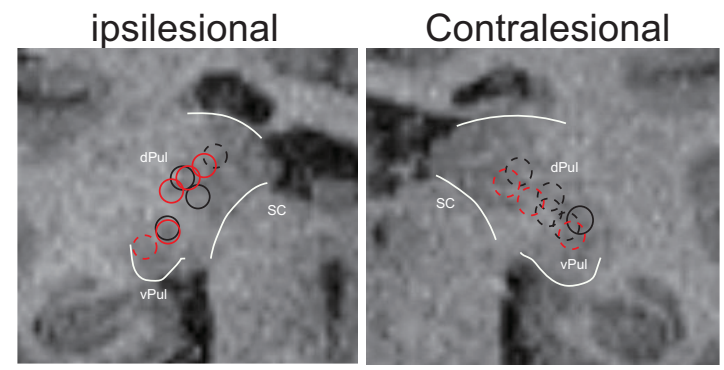

Figure 2. Injection sites of muscimol in the pulvinar and lateral geniculate nucleus. $\boldsymbol{A}$, The injection sites of muscimol in the pulvinar (Pul) are indicated as white shadows in the coronal planes of MR images. The right panel is the enlarged image of the area captured by the blue window in the left panel. Red arrow indicates the extent of the gadolinium shadow. The anteroposterior levels in stereotaxic coordinates are $+4.8 \mathrm{~mm}$. $\boldsymbol{B}$, The injection site of muscimol in the LGN. The same arrangement as $\boldsymbol{A}$. The anteroposterior levels in stereotaxic coordinates are $+8.8 \mathrm{~mm}$. $\boldsymbol{C}$, Injection sites in the Pul in each experimental session are indicated as a circle for the ipsilesional side (left) and for the contralesional side (right), respectively. Black circles are injection sites with significant inactivation effects in monkey 0 , and red circles are in monkey T. Dashed line indicates the location where the injection did not affect to the VGS performance. White lines indicate the border of Pul with the adjacent structures. The anteroposterior levels in the stereotaxic coordinates are $+4.8 \mathrm{~mm}$ for the left panel (ipsilesional side) and $+6.0 \mathrm{~mm}$ for the right panel (contralesional side).

Table 1. Number of affected sessions and STs by muscimol inactivation

\begin{tabular}{lllll}
\hline & Inactivation & $\begin{array}{l}\text { Affected } \\
\text { session }(n)\end{array}$ & $\begin{array}{l}\text { Affected } \\
\text { ST }(n)\end{array}$ & $\begin{array}{l}\text { Mean error rate } \\
\text { of affected STs }\end{array}$ \\
\hline Monkey 0 & ipsiPul & $4 / 6$ & $54 / 90$ & 0.8169 \\
& ipsiLGN & $6 / 6$ & $31 / 90$ & 0.546 \\
& contraPul & $1 / 6$ & $2 / 90$ & 0.9165 \\
Monkey T & contraLGN & $5 / 5$ & $27 / 75$ & 0.6804 \\
& ipsiPul & $7 / 8$ & $78 / 104$ & 0.7787 \\
& ipsiLGN & $5 / 5$ & $50 / 65$ & 0.7444 \\
& contraPul & $2 / 5$ & $2 / 75$ & 0.3136 \\
& contraLGN & $3 / 5$ & $8 / 75$ & 0.3736 \\
\hline
\end{tabular}

This table indicates how many sessions and STs were affected by muscimol injection. The left number in each cell shows the number of affected sessions or STs, and the right number shows the number of all sessions or all STs. Here, if the median of saccade amplitude exceeded the correct window (see Materials and Methods), we considered that the ST was affected by inactivation. If more than one ST was affected in a session, the session was defined as an affected session. The average trial number of each daily session ranged from 450 to 700 trials and from 510 to 1443 trials before and after muscimol injection, respectively. The average trial number for each ST location after muscimol injection ranged from 14 to 25. contra, Contralateral; ipsi, ipsilateral; LGN, lateral geniculate nucleus; Pul, pulvinar.

each other. The luminance Michelson contrast of the FP and ST was 0.95 (Weber contrast, 37.8) on a background of $1.0 \mathrm{~cd} / \mathrm{m}^{2}$. A water reward was delivered if the monkeys acquired the ST within $0.03 \mathrm{~s}$ after saccade initiation and stayed within the ST window for $800 \mathrm{~ms}$; otherwise, it was judged as an error trial. The error rate was calculated by dividing the number of error trials by the total number of trials (in which the fixation period was accomplished) during the 10 min block. If the median of the distances between the saccadic end points and STs was larger than the diameter of the correct window for each ST location, the performance of the saccade toward the ST location in the session was judged to be impaired by the muscimol injection (Table 1). The 
A

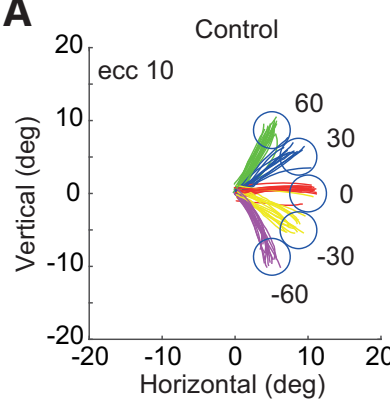

B

Control

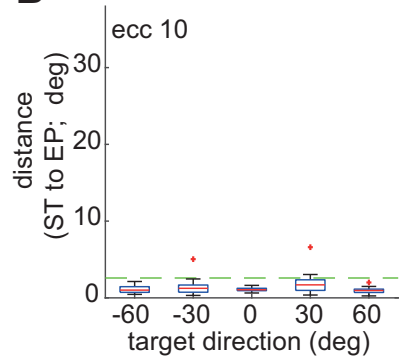

C $20.60 \mathrm{~min}$
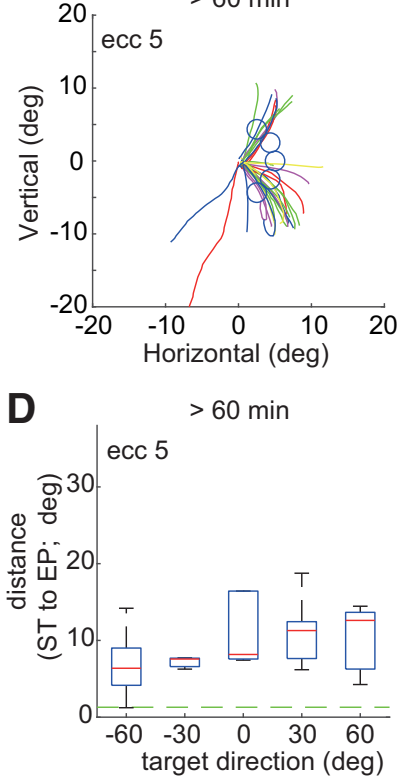

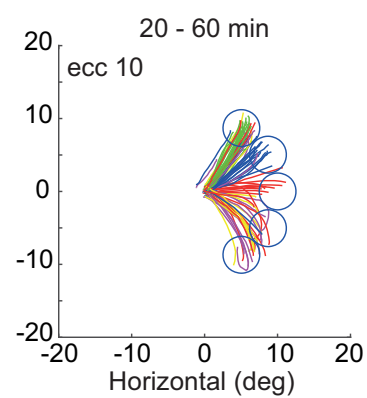

20 - 60 min
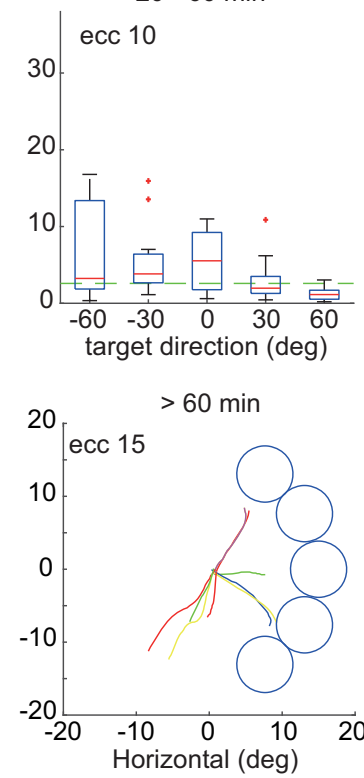

$>60 \mathrm{~min}$

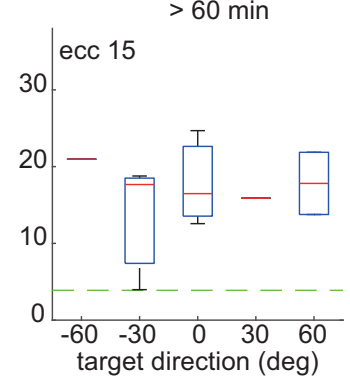

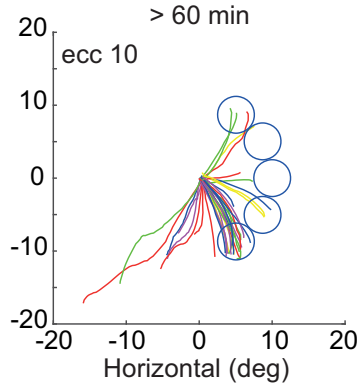

$>60 \mathrm{~min}$

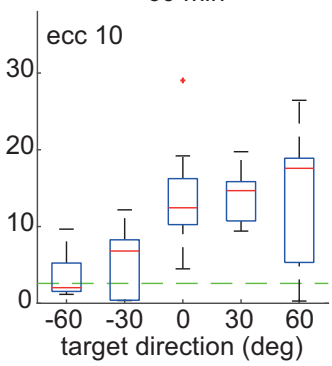

E

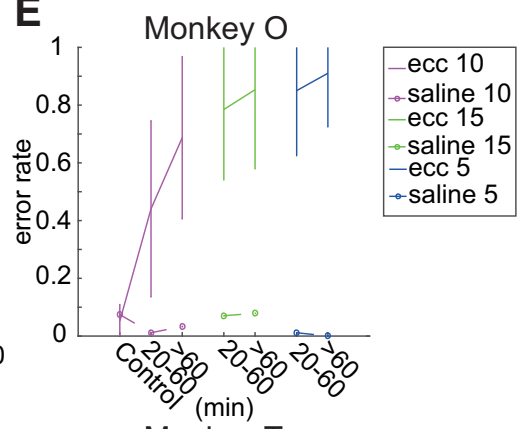

Monkey $\mathrm{T}$

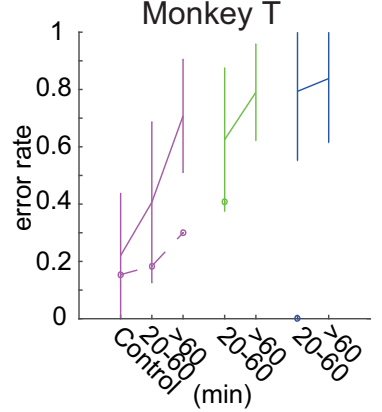

Figure 3. Effect of ipsilesional pulvinar inactivation on visually guided saccades. $\boldsymbol{A}-\boldsymbol{D}$, Typical examples were recorded in monkey 0 . $\boldsymbol{A}$, Saccadic trajectories before and after inactivation of the ipsilesional pulvinar. Control (left), at 20-60 min after injection (middle), and at $>60 \mathrm{~min}$ after injection (right). Eccentricity (ecc) of the STs was $10^{\circ}$. Each number in the left panel indicates the target direction. $\boldsymbol{B}$, Distance between the saccadic end points and STs before and after inactivation. Time from injection is indicated in the same manner as in $\boldsymbol{A}$. ST directions from $-60^{\circ}$ to $60^{\circ}$ correspond to those from low position to high position in $\boldsymbol{A}$. Horizontal green dashed lines indicate the error criteria. $\boldsymbol{C}$, Saccadic trajectories to STs with ecc of $5^{\circ}$ (left) and $15^{\circ}$ (right) during the period $>60 \mathrm{~min}$ after inactivation. $\boldsymbol{D}$, Distances between the saccade end points and STs for each direction of the ST with ecc of $5^{\circ}$ (left) and $15^{\circ}$ (right) after inactivation. $\boldsymbol{E}$, Error rates during the control and inactivation periods, and after injection of saline as a vehicle, for each ecc: $10^{\circ}$ (purple), $5^{\circ}$ (green), and $15^{\circ}$ (blue) are indicated for monkeys 0 (top) and T (bottom). Dashed lines with the corresponding color indicate the error rates for saline injections.

distribution of errors in direction and amplitude in Figure 3 (also see Figs. 7, 9) were calculated for these ST locations in these sessions.

We investigated the kinematics of eye movements by analyzing the relationship between the amplitude and the peak velocity of the saccades during the performance of the VGS task (main sequence). We used the following power model for fitting:

$$
f(x)=a \times\left(x^{b}\right),
$$

where, $f(x)$ and $x$ indicate saccade amplitude and saccade peak velocity, respectively. Here, the fitted parameter $a$ represents the slope value and $b$ represents the exponent value of the fitted curve. To assess whether the fitted parameters changed after inactivation of the LGN or pulvinar, we confirmed overlaps of the $95 \%$ confidence intervals.

Histologic processing. After the experiments were terminated (monkeys O, 6 months after V1 lesioning; monkey T, 50 months after V1 lesioning), the monkeys were anesthetized deeply with an intravenous injection of sodium pentobarbital $(50-100 \mathrm{mg} / \mathrm{kg})$ and transcardially perfused with $0.05 \mathrm{M}$ PBS and then with $4 \%$ paraformaldehyde in $0.1 \mathrm{M}$ phosphate buffer, $\mathrm{pH}$ 7.4. The brains were sectioned at a thickness 40 or $50 \mu \mathrm{m}$ in coronal slices. The LGN sections were immunostained with a calcium/calmodulin-dependent protein kinase II $\alpha$ (CaMKII $\alpha$ ) antibody (Affinity BioReagents) as described previously (Kinoshita et al., 2019). Adjacent sections were processed with $1 \%$ cresyl violet. 

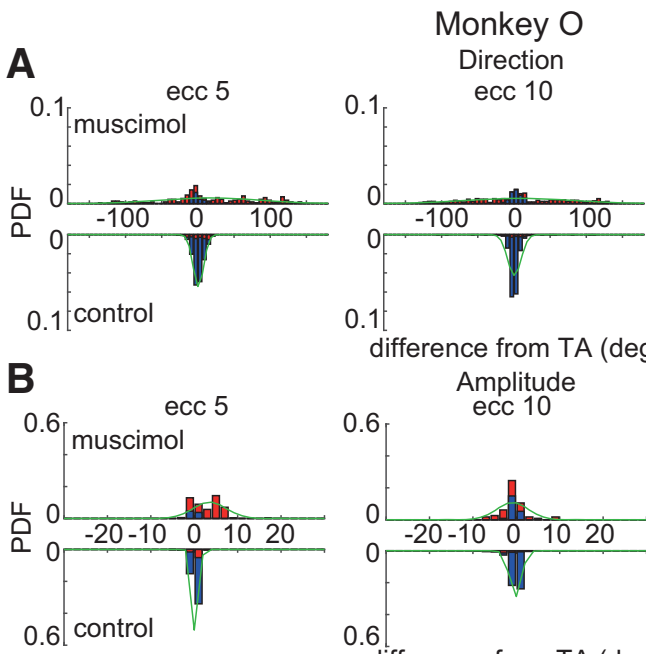

difference from TA (deg) Amplitude

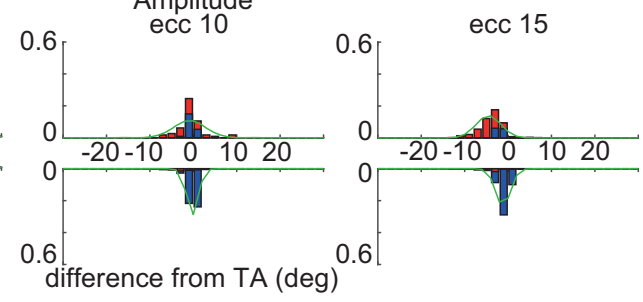

Monkey O
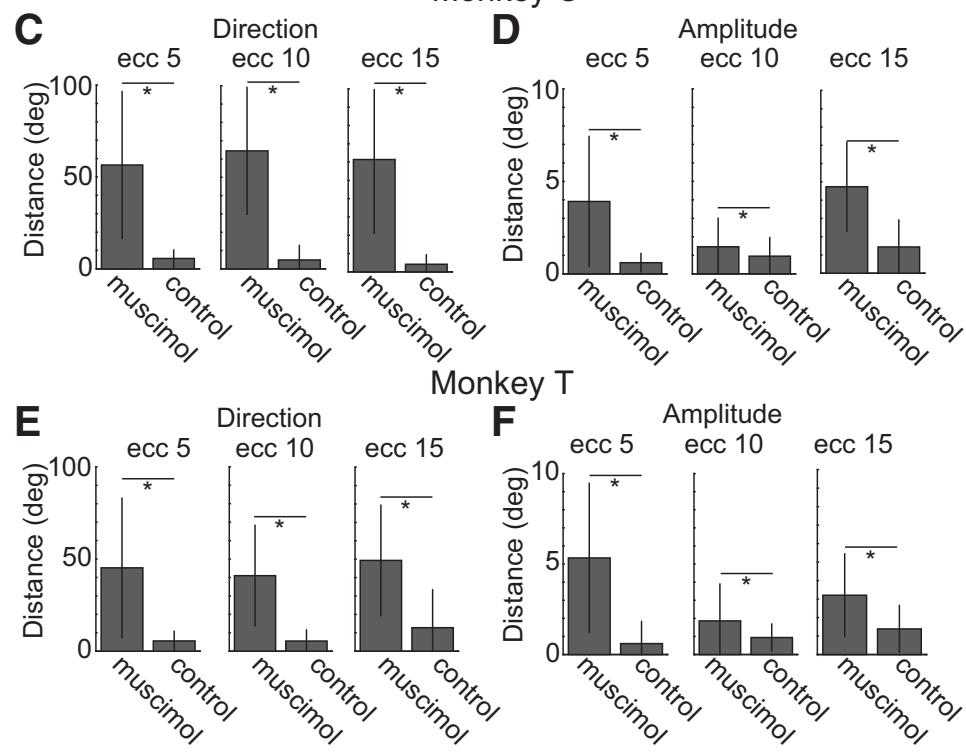

Figure 4. Direction and amplitude errors of visually guided saccades during the control period and after inactivation of the ipsilesional pulvinar. Direction and amplitude errors during the preinactivation (control) and inactivation periods are compared (monkey $0, N=558$; monkey $\mathrm{T}, N=1591$ ). $\boldsymbol{A}$, Distribution of saccade direction errors normalized by the probability density function (PDF). Data during the control and inactivation periods are indicated in the bottom and top panels, respectively. Correct trials are indicated in blue, while error trials are indicated in red. Green curved lines show Gaussian fitting of the distributions. ecc, Eccentricity. $\boldsymbol{B}$, Distribution of saccade amplitude errors. The same arrangement as in $\boldsymbol{A}$. $\boldsymbol{C}, \boldsymbol{D}$, Comparison of direction errors $(\boldsymbol{C})$ and amplitude errors $(\boldsymbol{D})$ between the control and inactivation periods in monkey 0 (C: two-sample $t$ test, *p>0.05; ecc $5^{\circ}, p=4.92 \times 10^{-34}$; ecc $10^{\circ}, p=2.53 \times 10^{-64}$; ecc $15^{\circ}, p=2.89 \times 10^{-48} ; \boldsymbol{D}$ : two-sample $t$ test, $* p>0.05$; ecc $5^{\circ}, p=1.02 \times 10^{-23}$; ecc $10^{\circ}, p=8.05 \times 10^{-6}$; ecc $\left.15^{\circ}, p=1.79 \times 10^{-46}\right) . \boldsymbol{E}, \boldsymbol{F}$, Comparison of direction errors $(\boldsymbol{E})$ and amplitude errors $(\boldsymbol{F})$ between the control and inactivation periods in monkey T. The same arrangements as $\boldsymbol{C}$ and $\boldsymbol{D}\left(\boldsymbol{E}\right.$ : two-sample $t$ test, $* p>0.05$; ecc $5^{\circ}$, $p=1.76 \times 10^{-82}$; ecc $10^{\circ}, p=4.61 \times 10^{-114}$; ecc $15^{\circ}, p=2.86 \times 10^{-99} ; \boldsymbol{F}$ : two-sample $t$ test, $* p>0.05$; ecc $5^{\circ}$, $p=8.94 \times 10^{-93} ;$ ecc $10^{\circ}, p=9.47 \times 10^{-22} ;$ ecc $\left.15^{\circ}, p=5.94 \times 10^{-55}\right)$.

Three coronal sections including one at the center of the LGN along the rostrocaudal axis and those at $1 \mathrm{~mm}$ rostral and caudal to it were selected, and the number of CaMKII $\alpha$-positive cells in these sections was counted as koniocellular neurons on the ipsilesional and contralesional sides as described previously (Kinoshita et al., 2019). Thus, the survival rate of koniocellular neurons was estimated by dividing the number of surviving cells on the ipsilesional side by the number on the contralesional side. For comparison, the data from three monkeys (C, A, and H) in our previous article (Kinoshita et al., 2019) were included in the data of this study.

To find the surviving neurons in LGN, the sections were immunohistochemically processed with the antibody against a neuronal marker,
NeuN. The sections were treated in $0.6 \%$ hydrogen peroxide in Dent's fixative followed by PBS with $10 \%$ normal goat serum (NGS) and $0.1 \%$ Triton $\mathrm{X}-100$ and reacted with mouse monoclonal antiNeuN antibody (1:400; Millipore) in PBS with $2 \%$ NGS and $0.1 \%$ Triton X-100 (PBST/NGS) at $4^{\circ} \mathrm{C}$ overnight. The sections were then incubated with biotin-conjugated anti-mouse IgG (1: 200; Invitrogen) in PBST/NGS followed by the incubation in an Invitrogen $\mathrm{ABC}$ Elite Kit (1:100; Thermo Fisher Scientific) and finally visualized with diaminobenzidine and nickel ammonium sulfate.

Experimental design and statistical analysis. Data were collected from two female Japanese monkeys. In each daily experiment, we inactivated the neural activity in the pulvinar or LGN with microinjection of muscimol. The injection was conducted more than five times for each brain area. After the inactivation, the monkeys had an interval day to wash out the effect of inactivation. The experiments were designed to compare the effects of inactivation of the pulvinar and LGN in the same monkey.

All statistical analyses were performed using the Statistics toolbox in MATLAB. Because of the experiments design, we used two-sample $t$ tests for the comparison of the control dataset with the dataset of inactivation experiments. The threshold for significance was set as $p<0.05$. The actual $p$ value for each analysis is described in the figure legends.

\section{Results}

Inactivation of the ipsilesional pulvinar

To investigate the role of the pulvinar in blindsight, we inactivated neural activity in the pulvinar ipsilateral to the V1 lesion by muscimol injection. Before inactivation, the monkeys were tested on their performance for VGS to five possible target locations with an eccentricity of $10^{\circ}$. In this case, their error rate was $<20 \%$ (Fig. 1D). As shown in Figure $2 C$, injection sites of muscimol covered a wide extent in the pulvinar, including its tectorecipient ventrolateral portion (Stepniewska et al., 1999). Inactivation of the pulvinar impaired their performance. They made saccades in the wrong direction or with the wrong amplitude (Fig. 3A), or their gaze remained on the FP and did not move even after target presentation. For example, in the case shown in Figure $3 A$, the saccade amplitudes appeared to be correct, but the saccade direction became scattered for saccades toward the ST in the $0^{\circ}$ and $-30^{\circ}$ directions between 20 and $60 \mathrm{~min}$ after injection (Fig. $3 A$, middle). At $60 \mathrm{~min}$ after injection, the monkey often chose the ST in the $-60^{\circ}$ direction, presumably with guessing, even if saccades were required to other ST locations (Fig. 3A, right). The error patterns were not consistent among the daily sessions. The medians of the distances between the saccade end points and ST locations were mostly within the correct window indicated by the green dashed lines before inactivation, as shown in Figure $3 B$ (left); 
however, they clearly increased beyond the window after inactivation (Fig. 3B). Muscimol injection affected saccades toward a wide range (four of five targets) of the visual field on the side contralateral to the injection at $>60 \mathrm{~min}$ after injection. The targets were also presented at eccentricities of $5^{\circ}$ and $15^{\circ}$ from the FP in different experimental blocks. The impairment of saccade performance was also observed in all five directions during the $5^{\circ}$ and $15^{\circ}$ blocks (Fig. 3C,D). The error rate was higher than during the control period (Fig. 3E; two-sample $t$ test, $p<0.05)$. In the case of vehicle (saline) injections, the medians of the distance between the saccade end points and ST locations were smaller than the diameter of the ST window for all eccentricities in both monkeys. These results confirmed the observations in our previous study (Kinoshita et al., 2019). In the present study, we did not test VGSs to the ST in the ipsilesional visual field, except at an eccentricity of $10^{\circ}$, because we reported previously that pulvinar inactivation had no effect on VGS toward the ipsilesional visual field (Kinoshita et al., 2019). In each experiment, we injected muscimol at one location among a variety of locations inside the pulvinar, and the amount was relatively small $(0.5 \mu \mathrm{l}, 1.0 \mu \mathrm{g} / \mu \mathrm{l})$. However, the injection caused a clear deficit in saccades toward a wide area of the contralateral visual field, maybe because the retinotopic map of the pulvinar is less clear and the response field of each pulvinar neuron in primates covers a wider visual field than the neurons in other regions of the visual thalamus such as the LGN, as suggested by previous studies (Gattass et al., 1978; Bender, 1981; Li et al., 2013). Thus, as shown in Table 1, impairment of saccades was observed for 54 ST locations (among a total of 90 ST locations) in all six daily sessions in monkey $\mathrm{O}$ and for 78 ST locations (among a total of 104 ST locations) in seven daily sessions in monkey $\mathrm{T}$ for ipsilesional pulvinar inactivation.

To clarify the pattern of saccadic errors after pulvinar inactivation, we divided the errors into amplitude error and directional error for all saccades toward the ST locations to which the saccades were judged to be impaired (Fig. $4 A, B$ ). We found that inactivation of the pulvinar caused errors in amplitude and direction. The direction of error saccades was distributed in a wide range. In contrast, the amplitude errors were relatively small in the case of a $10^{\circ}$ block. However, saccade amplitude shifted to a $10^{\circ}$ eccentricity in the case of $5^{\circ}$ and $15^{\circ}$ blocks. Such a trend might have been caused by our task design. Our task always started from the $10^{\circ}$ eccentricity block. Therefore, it is likely that the monkeys continued to make saccades to $10^{\circ}$ eccentricity after pulvinar inactivation in an internally driven manner, presumably based on
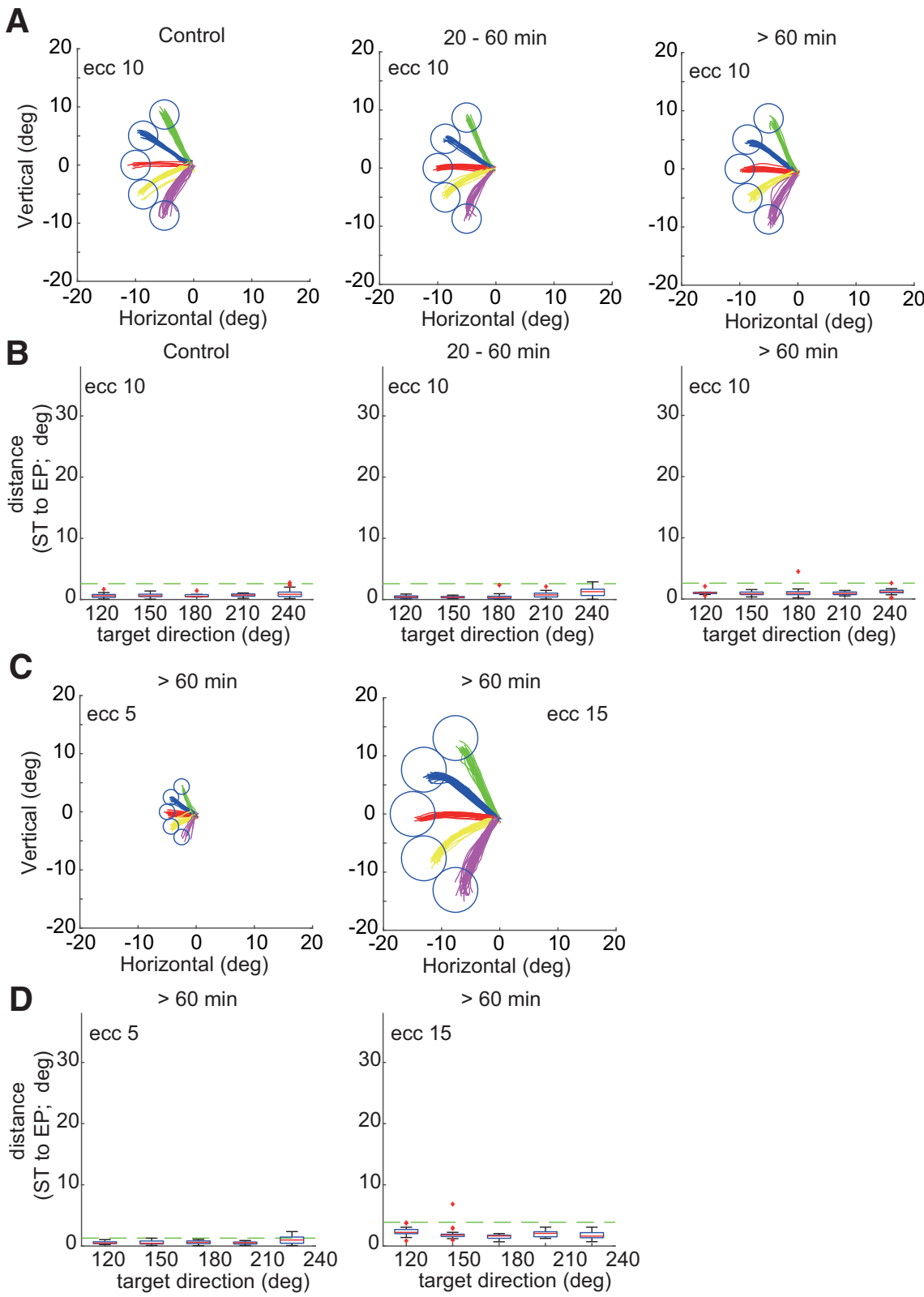

Figure 5. Effect of contralesional pulvinar inactivation on visually guided saccades. Typical examples in $\boldsymbol{A}-\boldsymbol{D}$ were recorded with monkey 0. A, Saccadic trajectories before and after inactivation of the contralesional pulvinar. Control (left), 20-60 min after muscimol injection (middle), and $>60$ min after injection (right). Eccentricity (ecc) of the STs was $10^{\circ}$. $\boldsymbol{B}$, Distance between saccade end points and STs before and after inactivation. Time from injection is indicated in the same manner as $\boldsymbol{A}$. Horizontal deen dashed lines indicate the error criteria. C, Saccadic trajectories to STs with ecc $5^{\circ}$ (left) and $15^{\circ}$ (right) during the period $>60$ min after inactivation. D, Distance between saccade end points and STs for each direction of ST with ecc $5^{\circ}$ (left) and $15^{\circ}$ (right) after inactivation. EP, End point.

experience or anticipation. These results suggest that ipsilesional pulvinar inactivation impaired the visual input stage rather than the visuomotor processing stage through the control of VGSs.

\section{Inactivation of the contralesional pulvinar}

To investigate the role of the pulvinar with an intact V1, we injected muscimol into the contralesional pulvinar. In this case, we did not observe a clear deficit in VGS. Typical examples of saccadic trajectories toward the target eccentricities of $10^{\circ}$ (Fig. $5 A$ ), $5^{\circ}$ (Fig. $5 C$ ), and $15^{\circ}$ (Fig. $5 C$ ) are shown in Figure 5 . The medians of the distances between the saccade end points and ST were less than the diameter of the correct window, in all cases for all eccentricities in the daily session shown in Figure 5, $B$ 
and $D$. In total, as shown in Table 1 , the performance of saccades was affected in 2 of 90 ST locations across a total of six daily sessions in monkey $\mathrm{O}$ and in 2 of 75 ST locations across a total of five daily sessions in monkey $\mathrm{T}$ for contralesional pulvinar inactivation. The two ST locations for which saccade performance was impaired in monkey $\mathrm{O}$ were both from a single daily session in which a relatively larger dose $(2.0 \mu \mathrm{l}, 1.0 \mu \mathrm{g} / \mu \mathrm{l})$ of muscimol was injected into the most rostral track of the pulvinar. It was considered that this effect was caused by the diffusion of muscimol into the LGN. Regarding monkey $\mathrm{T}$, the performance of saccades to the $5^{\circ} \mathrm{ST}$ was not accurate even in the control sessions (Fig. 1D), and there was no significant difference in the medians of the distances between the saccade end points and STs between the control and inactivation sessions toward these two ST locations. Therefore, we concluded that inactivation of the contralesional pulvinar did not affect the ability of blindsight.

Conversely, we found another deficit caused by inactivation of the contralesional pulvinar. After inactivation, the monkeys' eyes shifted to the side contralateral to the injection and it became difficult for the animals to maintain fixation on the FP (Fig. 6). However, even with such a deficit, as long as the ST was displayed while the animals were maintaining fixation and they could initiate saccades to the ST, they could correctly perform saccades. This phenomenon was observed in both monkeys (monkey $\mathrm{O}$, one of six daily sessions; monkey $\mathrm{T}$, two of seven daily sessions) when inactivating the contralesional pulvinar, but not when inactivating the ipsilesional pulvinar. The data from these two daily sessions in monkey $\mathrm{T}$ were removed from the analysis described above, because the animal lost motivation and stopped performing the task during the early period of the sessions. Here, it has been reported that inactivation of the pulvinar causes neglect in the contralateral visual hemifield (Wilke et al., 2010). In this study, however, this aspect was unclear after inactivation of the contralesional pulvinar, because we used only single-target stimuli in the VGS task. Deviation of eye positions during the free viewing was not noticed. Together, these results suggest that when $\mathrm{V} 1$ is intact, the pulvinar contributes to gaze fixation, but does not contribute directly to visual processing for saccade control.

Thus, the effects of inactivation of the ipsilesional and contralesional pulvinar were clearly different. This difference suggested the possibility that the roles of pulvinar in blindsight were derived from plastic changes in the visual pathways involving the pulvinar after V1 lesioning.

\section{Inactivation of the ipsilesional LGN}

To investigate the role of the LGN in the same monkeys used in the pulvinar inactivation experiments, we injected muscimol into the ipsilesional LGN. We predicted that the pulvinar, but not the LGN, plays the key role in blindsight with an extensive V1 lesion, because our previous study revealed that V1 lesioning induced considerable degeneration of the LGN and the number of koniocellular neurons decreased to $18-34 \%$ (compared with the intact side) at 3-8 years after lesioning (Kinoshita et al., 2019). However, in the present study, inactivation of the ipsilesional LGN affected the performance of VGSs in monkey O (at 1-5 months after lesioning) and monkey $\mathrm{T}$ (at 4047 months after lesioning; Fig. $7 A$ ). Their performance in the VGS task was impaired and the muscimol-affected visual field covered the eccentricities from $5^{\circ}$ to $15^{\circ}$ (Fig. $7 \mathrm{C}$ ). The distance between the saccadic end points and ST
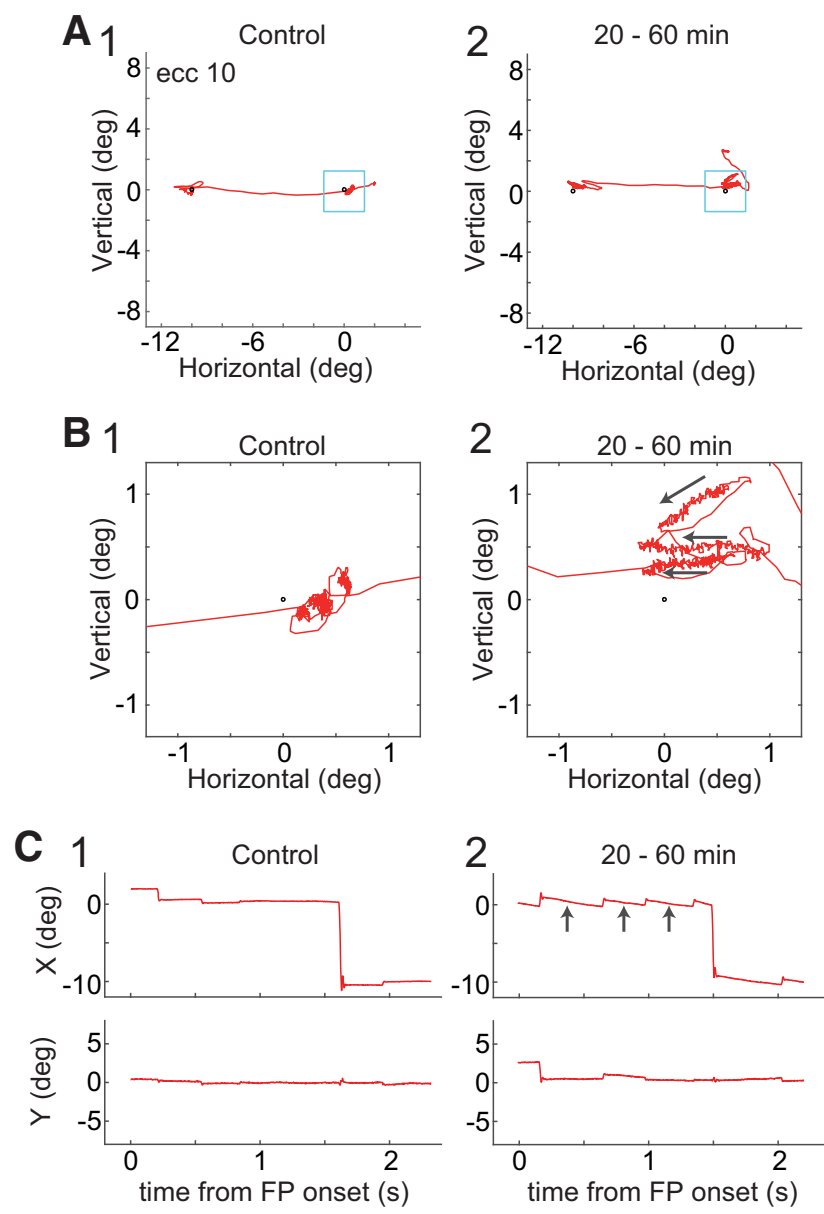

Figure 6. Effect of contralesional pulvinar inactivation on gaze fixation. $A$, Examples of gaze trajectory during a VGS task (red line). A black point in the blue square indicates the location of the FP, and another black point on the left indicates the location of the ST. A1,2, A trial during the control period (1) and another trial during the period 20-60 min after muscimol injection (2). ecc, Eccentricity. $\boldsymbol{B}$, Enlarged gaze trajectories within the blue boxes in $\boldsymbol{A} . \mathbf{B 1}, \mathbf{2}$, An example during the control period (1), and an example during the inactivation period (2). Black arrows indicate the direction of involuntary gaze movements. $C$, Horizontal ( $X$; top panels) and vertical ( $Y$; bottom panels) positions of gaze during the VGS task including fixation on the FP, saccade, and fixation on the ST shown in $\boldsymbol{A}, \mathbf{1}$ and $\mathbf{2}$. Black arrows in $\mathbf{C}$ indicate involuntary gaze movements during fixation.

became longer than the error criteria, especially at $60 \mathrm{~min}$ after injection (Fig. $7 B, D$ ). During this time period, the error rate for an eccentricity of $10^{\circ}$ was higher than during the control period (Fig. $7 E$; two-sample $t$ test, $p<0.05$ ). In the saline injection experiments $(N=2)$, the medians of the distance between the saccade end points and ST locations were smaller than the diameter of the ST windows.

To investigate the error components of saccades after ipsilesional LGN inactivation, we compared the errors in saccade direction and amplitude with the control data. The errors of direction had a wider distribution than in the control data (Fig. $8 A$ ), and the errors of amplitude appeared to be caused by the tendency of the monkeys to make $10^{\circ}$ saccades (Fig. $8 B$ ), similar to those for ipsilesional pulvinar inactivation described above. The differences in saccadic direction and amplitude from the ST were significantly larger after inactivation of the ipsilesional LGN than in the control data (Fig. $8 C-F$ ). As shown in Table 1, a significant effect was observed in all six daily sessions (31 of 90 ST locations) in monkey $\mathrm{O}$ and in all five daily sessions (50 of 65 ST 
A

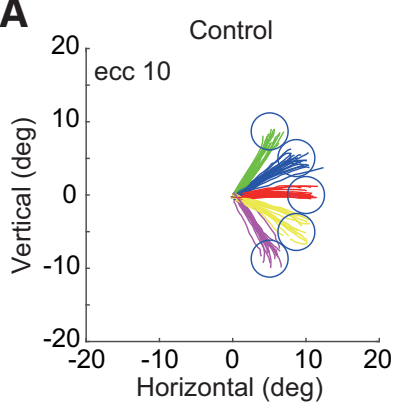

B

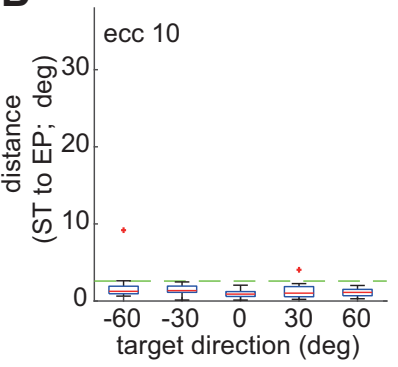

C
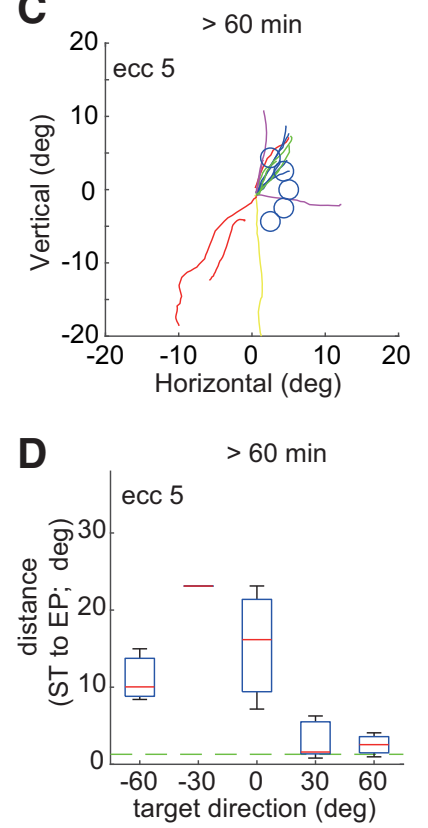

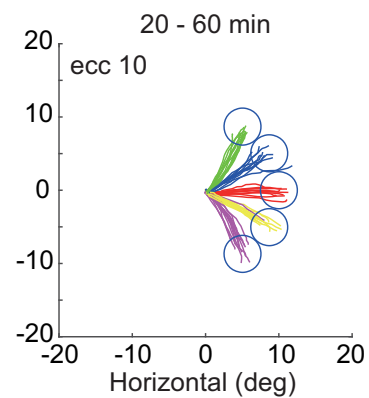

$20-60 \min$
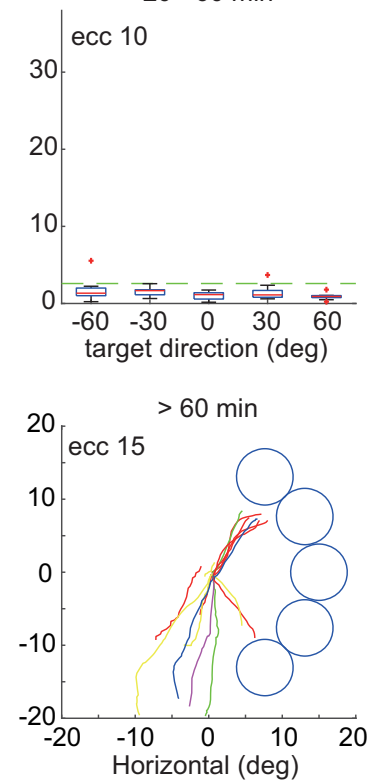

\section{E Monkey O}
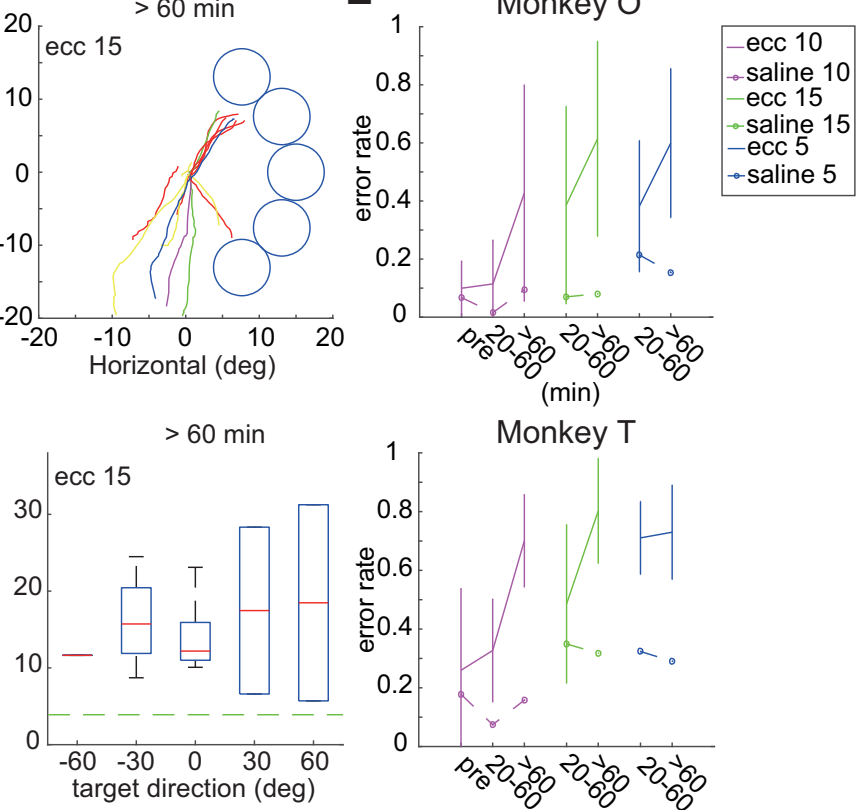

Monkey $\mathrm{T}$

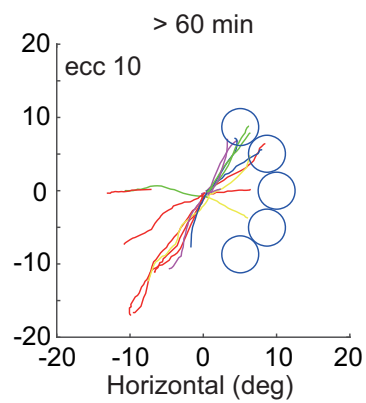

$>60 \mathrm{~min}$
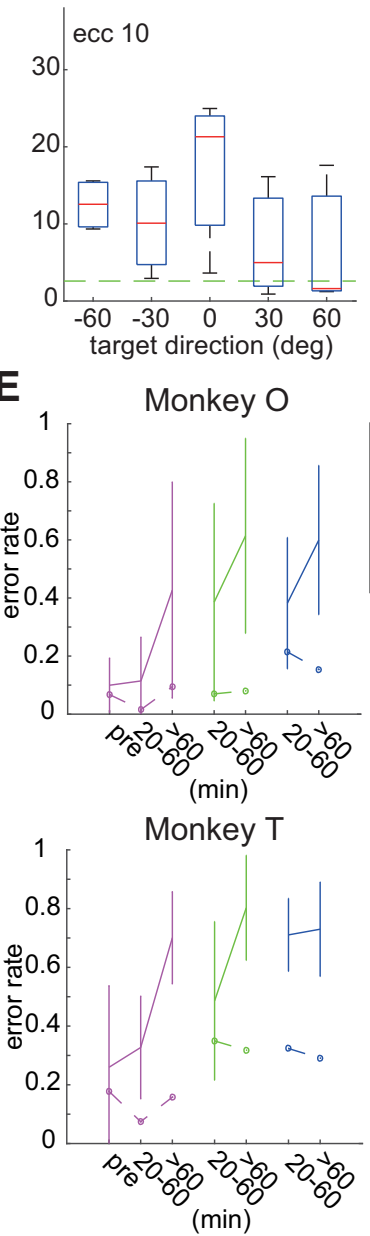

Figure 7. Effect of ipsilesional LGN inactivation on visually guided saccades. Typical examples in $\boldsymbol{A}-\boldsymbol{D}$ were recorded with monkey 0 . $\boldsymbol{A}$, Saccadic trajectories before and after inactivation of the ipsilesional LGN. Control period (left), 20-60 min after muscimol injection (middle), and $>60$ min after injection (right). Eccentricity (ecc) of the STs is $10^{\circ}$. B, Distance between the saccade end points and STs before and after inactivation. Time from injection is indicated in the same manner as in $A$. Horizontal green dotted lines indicate the error criteria. EP, End point. C, Saccadic trajectories to STs with ecc of $5^{\circ}$ (left) and $15^{\circ}$ (right) during the period $>60$ min after inactivation. D, Distance between the saccade end points and STs for each ST location with ecc of $5^{\circ}$ (left) and $15^{\circ}$ (right) after inactivation. $\boldsymbol{E}$, Error rates during the control and inactivation periods, and after saline injection as a vehicle, for each ecc: $10^{\circ}$ (purple), $5^{\circ}$ (green), and $15^{\circ}$ (blue) are indicated for monkeys 0 (top) and $\mathrm{T}$ (bottom). Dashed lines with the corresponding color indicate the error rates for saline injection.

locations) for ipsilesional LGN inactivation. These data suggested that the LGN is necessary for blindsight after damage to almost the entire V1.

\section{Inactivation of the contralesional LGN}

To confirm the role of the LGN on the side with the intact V1, we injected muscimol into the contralesional LGN. Inactivation of the contralesional LGN also induced a deficit in VGS performance (Fig. 9A,B). This effect started to appear at 20-60 min after injection. The error rate of the VGS task was significantly higher at an eccentricity of $10^{\circ}$ than in the control period and after vehicle (saline) injection (Fig. 9E). Regarding error analysis, differences in saccadic direction and amplitude from ST relative to the FP was significantly larger after inactivation of the contralesional LGN than in the control (Fig. 10), except for amplitude errors in the trials for an eccentricity of $10^{\circ}$ in monkey $\mathrm{O}$, presumably because the animals were primarily trained to make $10^{\circ}$ saccades (Fig. 10D). As shown in Table 1, a significant effect was observed in 

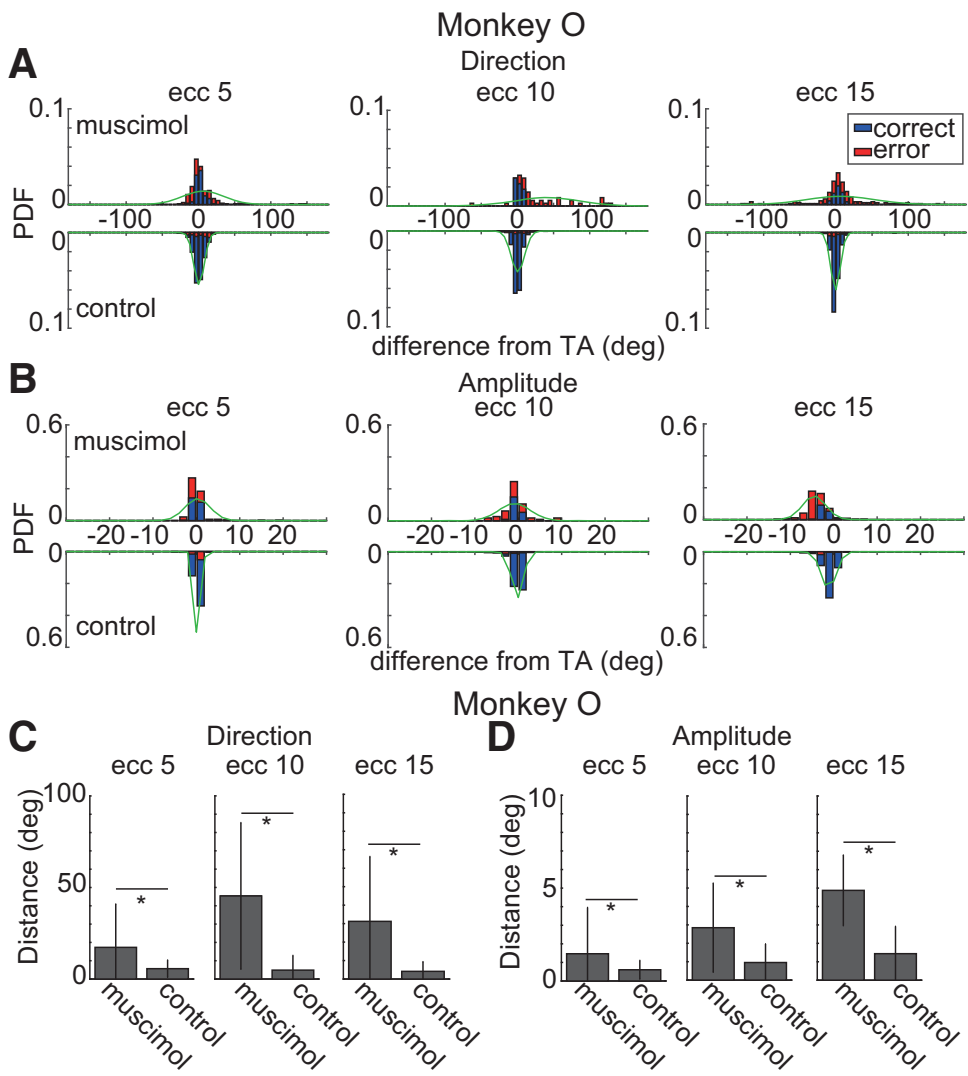

Monkey O
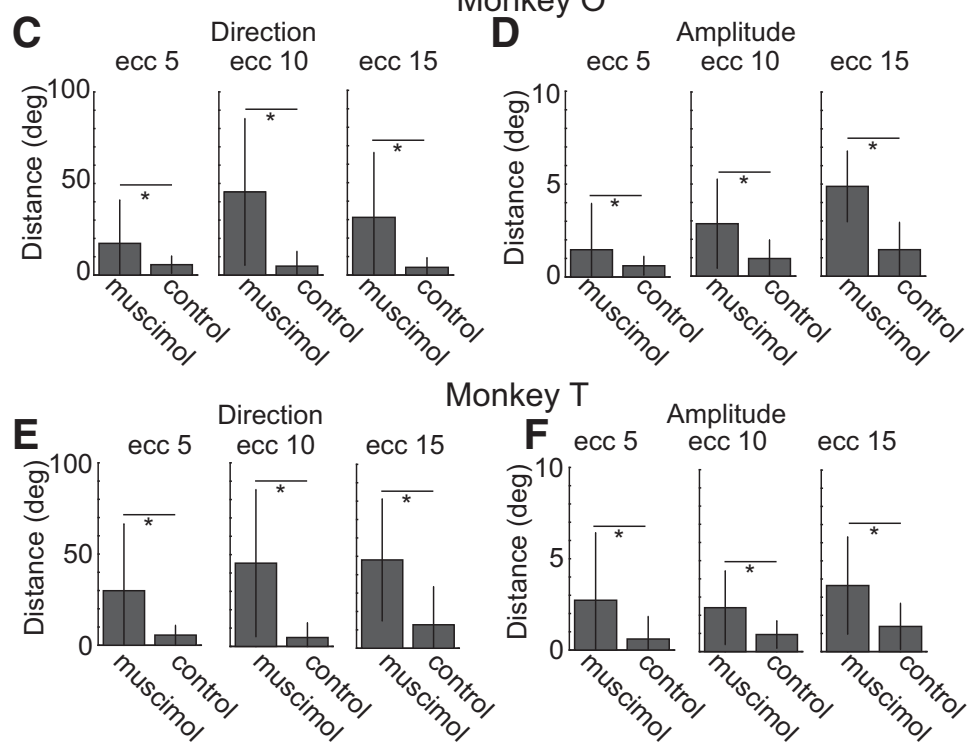

Figure 8. Direction and amplitude errors of VGS during the control period and after ipsilesional lateral geniculate nucleus inactivation. To assess the property of saccade errors, the direction and amplitude of VGS were compared during the control and inactivation periods (monkey $0, N=266$; monkey $\mathrm{T}, N=899$ ). $\boldsymbol{A}$, Distribution of saccade direction errors normalized by the probability density function (PDF). Data during the control and inactivation periods are indicated in the bottom and top panels, respectively. Correct trials are indicated in blue, while error trials are indicated in red. Green curved lines show Gaussian fitting of the distributions. ecc, Eccentricity. B, Distribution of saccade amplitude errors. The same arrangement as in $\boldsymbol{A}$. $\boldsymbol{C}, \boldsymbol{D}$, Comparison of direction errors $(\boldsymbol{C})$ and amplitude errors $(\boldsymbol{D})$ between the control and inactivation periods in monkey 0 (C: two-sample $t$ test, $* p>0.05$; ecc $5^{\circ}, p=6.67 \times 10^{-7}$; ecc $10^{\circ}, p=1.87 \times 10^{-6}$; ecc $15^{\circ}, p=1.38 \times 10^{-13} ; \mathbf{D}$ : two-sample $t$ test, $* p>0.05$; ecc $5^{\circ}, p=3.19 \times 10^{-4}$; ecc $10^{\circ}, p=5.45 \times 10^{-5}$; ecc $\left.15^{\circ}, p=9.86 \times 10^{-40}\right) . \boldsymbol{E}, \boldsymbol{F}$, Comparison of direction errors $(\boldsymbol{E})$ and amplitude errors $(\boldsymbol{F})$ between the control and inactivation periods in monkey $\mathrm{T}$, with the same arrangements as in $\boldsymbol{C}$ and $\boldsymbol{D}\left(\boldsymbol{E}\right.$ : two-sample $t$ test, $* p>0.05$; ecc $5^{\circ}$, $p=5.72 \times 10^{-33}$; ecc $10^{\circ}, p=5.02 \times 10^{-26} ;$ ecc $15^{\circ}, p=2.73 \times 10^{-49} ; \boldsymbol{F}$ : two-sample $t$ test, $* p>0.05$; ecc $5^{\circ}$, $p=2.43 \times 10^{-25} ;$ ecc $10^{\circ}, p=3.86 \times 10^{-20} ;$ ecc $\left.15^{\circ}, p=1.44 \times 10^{-35}\right)$.

all five daily sessions (27 of 75 locations) in monkey $\mathrm{O}$ and in three of five daily sessions (8 of 75 ST locations) for contralesional LGN inactivation. The extent of the ST locations affected by the contralesional LGN was more limited than during inactivation of the pulvinar; however, the effect was profound, as shown in Figure 10. These results suggest that the LGN is needed for VGS with an intact V1.

\section{Kinematics of saccades after inactivation of the ipsilesional pulvinar and LGN}

To investigate whether the kinematics of VGS were affected by inactivation of the ipsilesional pulvinar and LGN, the relationship between the peak velocity and amplitude of VGS (main sequence relationship) was examined before and after inactivation of the ipsilesional pulvinar and LGN (Fig. 11). All saccades, both correct and error, during the trials were collected for analysis. As shown in Figure 11, analysis of the main sequence relationship revealed a slight, but significant, decrease in saccade velocity after inactivation of the ipsilesional LGN from the control in monkey $\mathrm{O}$ (see Materials and Methods; Fig. 11A, asterisk). There was no significant difference in the other conditions, for control versus inactivation of the ipsilesional pulvinar in monkey $\mathrm{O}$, and for both conditions in monkey $\mathrm{T}$.

\section{Evaluation of the retrograde degeneration of LGN neurons}

Our previous study showed that at 40101 months after lesioning, the magnocellular and parvocellular LGN neurons were mostly degenerated and that only $18-34 \%$ of koniocellular neurons remained (Kinoshita et al., 2019). The present results suggested that the LGN contributes to VGS performance in blindsight monkeys during the early (monkey O, 1-5 months) and late (monkey T, 4047 months) periods after V1 lesioning. To evaluate the retrograde degeneration and survival of neurons in the LGN, the ipsilesional and contralesional LGNs of both monkeys were processed for Nissl staining and anti-NeuN immunostaining to observe the survival of LGN neurons in general, and anti-CaMKII immunostaining was used to visualize the koniocellular neurons. In monkey O (Fig. 12A,C,E), with Nissl staining, it was difficult to find the surviving cells in the lesion-affected area of LGN as in our previous study (Kinoshita et al., 2019; Fig. $12 A 1,3)$. However, with anti-NeuN immunostaining, a small number of neurons was found to survive in the lesion-affected area (Fig. 12C3). Furthermore, antiCaMKII $\alpha$ immunostaining revealed surviving koniocellular neurons (Fig. 12E1, $3,5)$. The survival rate of koniocellular neurons on the ipsilesional LGN was $52 \%$ (969 of 1853) compared with the corresponding area of the contralesional LGN (Fig. 12E). In monkey $\mathrm{T}$, the survival rate of koniocellular neurons on the ipsilesional LGN compared with those on the contralesional LGN was $35 \%$ ( 824 of 2324; Fig. 12F). The surviving neurons could be detected by anti-NeuN immunostaining (Fig. 12D3) as well as in monkey $\mathrm{O}$ (Fig. 12C3). In both monkeys, koniocellular neurons survived both in the magnocellular domain (K1-K2; Fig. 12E3,F3) and in the parvocellular domain (K3-K6; Fig. 12E5,F5). Comparing the number of surviving cells detected by anti-NeuN and anti-CaMKII $\alpha$ staining, it was not likely that all the surviving cells were koniocellular. Thus, massive retrograde degeneration of the 
A

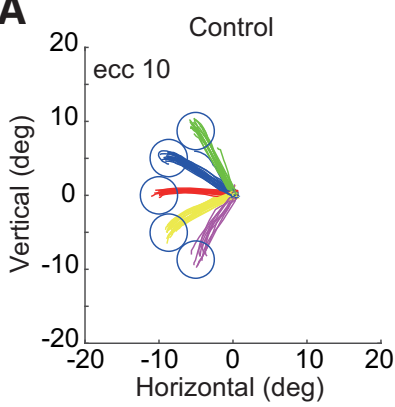

B

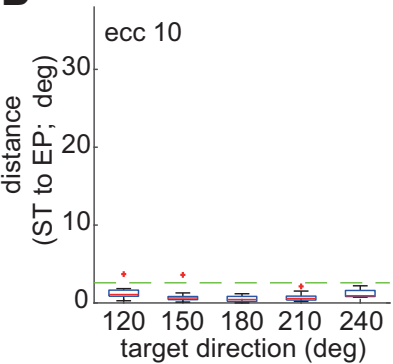

C
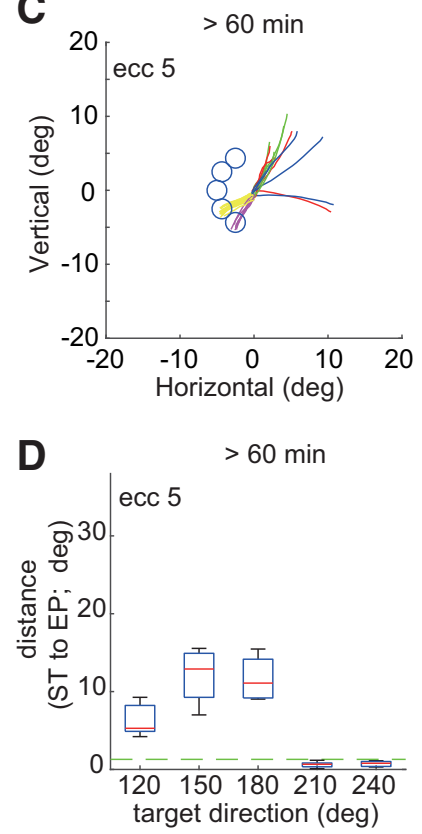

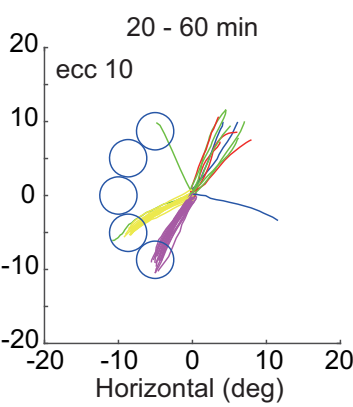

$20-60 \mathrm{~min}$
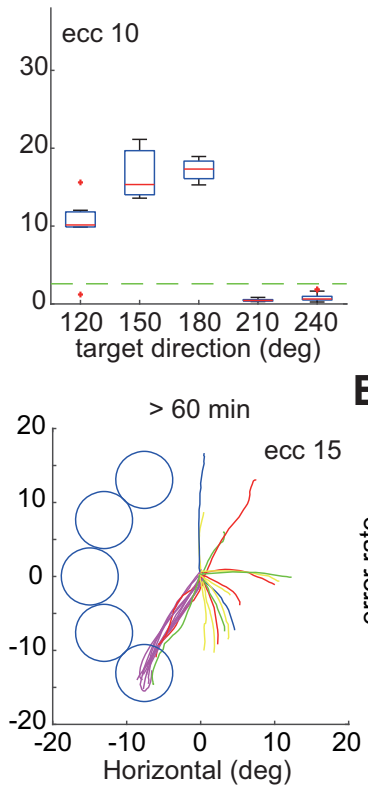

$>60 \mathrm{~min}$

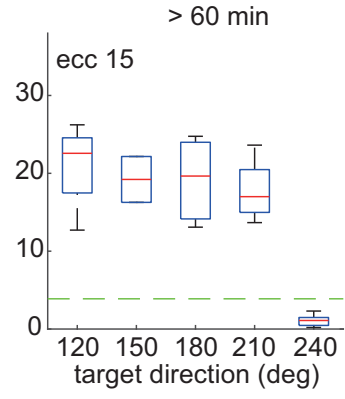

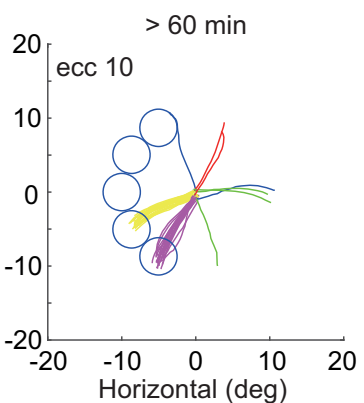

$>60 \mathrm{~min}$

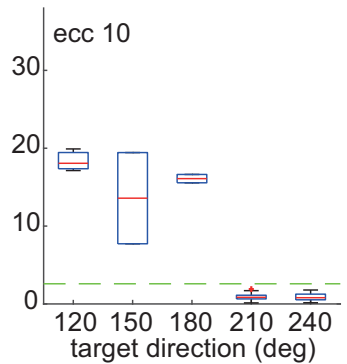

E

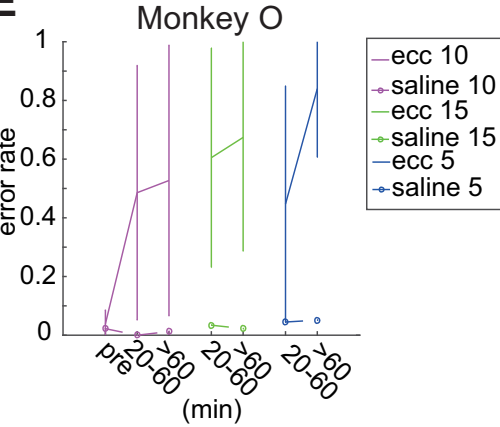

Monkey $\mathrm{T}$

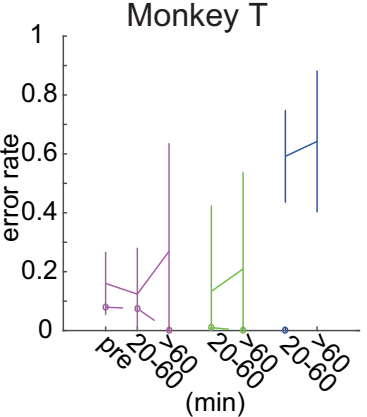

Figure 9. Effect of contralesional LGN inactivation on visually guided saccades. Typical examples in $\boldsymbol{A}-\boldsymbol{D}$ were recorded with monkey 0 . $\boldsymbol{A}$, Saccadic trajectories before and after inactivation of the ipsilesional LGN. Control period (left), 20-60 min after muscimol injection (middle), and $>60$ min after injection (right). Eccentricity (ecc) of the STs was $10^{\circ}$. $\boldsymbol{B}$, Distance between the saccade end points and STs before and after inactivation. The time from injection is indicated in the same manner as in $\boldsymbol{A}$. Horizontal green dashed lines indicate the error criteria. EP, End point. C, Saccadic trajectories to STs with ecc of $5^{\circ}$ (left) and $15^{\circ}$ (right) during the period $>60$ min after inactivation. D, Distances between the saccade end points and STs for each ST location with ecc of $5^{\circ}$ (left) and $15^{\circ}$ (right) after inactivation. $\boldsymbol{E}$, Error rates during the control and inactivation periods, and after injection of saline as a vehicle, for each ecc: $10^{\circ}$ (purple), $5^{\circ}$ (green), and $15^{\circ}$ (blue) are indicated for monkey 0 (top) and monkey $\mathrm{T}$ (bottom). Dashed lines with the corresponding color indicate the error rates for saline injection.

magnocellular and parvocellular neurons was confirmed in the ipsilesional LGN; however, it was likely that a small portion of magnocellular and parvocellular LGN neurons survived together with the koniocellular neurons as previously shown in marmosets (Atapour et al., 2017; Yu et al., 2018) and in macaques (Matthews et al., 1960). Figure $12 G$ shows the relationship between the survival time of the monkeys $(\mathrm{O}, \mathrm{C}$, $\mathrm{T}, \mathrm{A}$, and $\mathrm{H}$ ) after $\mathrm{V} 1$ lesioning and the survival rate of koniocellular neurons (the survival time and survival rate of koniocellular neurons were 40 months and $34 \%$ for monkey C,
71 months and $22 \%$ for monkey A, and 101 months and $18 \%$ for monkey H, respectively; Kinoshita et al., 2019). As shown in Figure 12G, considerable retrograde degeneration had occurred before 6 months after V1 lesioning and the number of koniocellular neurons gradually reduced over time after injury; however, even at $>8$ years after lesioning, $18 \%$ of neurons still survived. This could explain the contribution of the LGN to saccade control even long after V1 lesioning. In contrast to LGN, degeneration was not clear in the pulvinar (data not shown). 

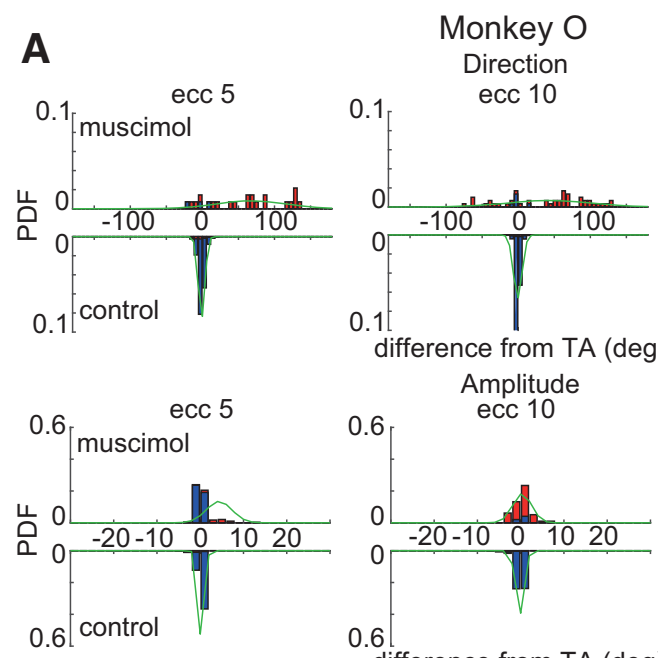

Amplitude

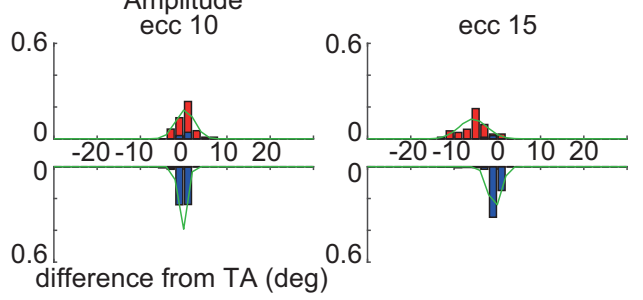

Monkey O
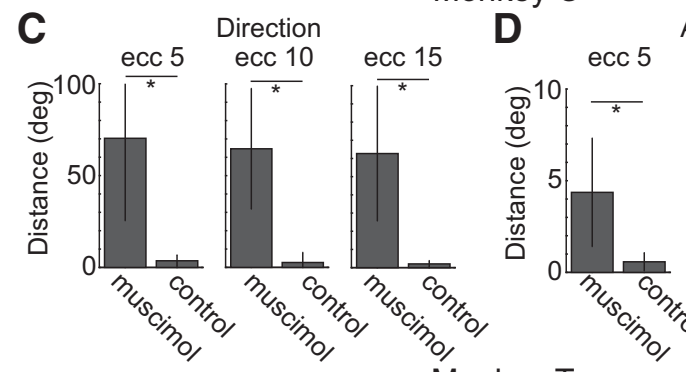

Amplitude

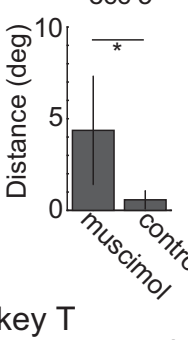

Monkey T
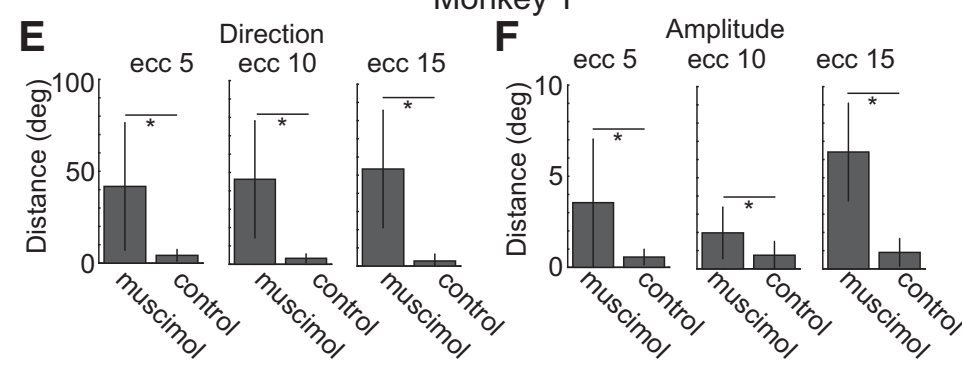

Figure 10. Direction and amplitude errors of VGSs during the control period and after inactivation of the contralesional lateral geniculate nucleus. To assess the property of saccade errors, the direction and amplitude of VGSs were compared during the control and inactivation periods (monkey $0, N=110$; monkey $\mathrm{T}, N=157$ ). $\boldsymbol{A}$, Distribution of saccade direction errors normalized by the probability density function (PDF). Data during the control and inactivation periods are indicated in the bottom and top panels, respectively. Correct trials are indicated in blue, while error trials are indicated in red. Green curved lines show Gaussian fitting of the distributions. ecc, Eccentricity. $\boldsymbol{B}$, Distribution of saccade amplitude errors. The same arrangement as in $\boldsymbol{A}$. $\boldsymbol{C}, \boldsymbol{D}$, Comparison of direction errors $(\boldsymbol{C})$ and amplitude errors $(\boldsymbol{D})$ in the control and inactivation periods in monkey 0 (C: two-sample $t$ test, $* p>0.05$; ecc $5^{\circ}, p=2.28 \times 10^{-6}$; ecc $10^{\circ}, p=1.27 \times 10^{-15}$; ecc $15^{\circ}$, $p=8.51 \times 10^{-15} ; \boldsymbol{D}$ : two-sample $t$ test, $* p>0.05$; ecc $5^{\circ}, p=1.56 \times 10^{-5}$; ecc $10^{\circ}, p=1.46 \times 10^{-4}$; ecc $15^{\circ}$, $\left.p=2.15 \times 10^{-14}\right) . \boldsymbol{E}, \boldsymbol{F}$, Comparison of direction errors $(\boldsymbol{E})$ and amplitude errors $(\boldsymbol{F})$ between the control and inactivation periods in monkey T. The same arrangements as in $\boldsymbol{C}$ and $\boldsymbol{D}\left(\boldsymbol{E}\right.$ : two-sample $t$ test, $* p>0.05$; ecc $5^{\circ}, p=6.17 \times 10^{-11}$; ecc $10^{\circ}, p=3.80 \times 10^{-11}$; ecc $15^{\circ}, p=4.09 \times 10^{-17} ; \boldsymbol{F}$ : two-sample $t$ test, $* p>0.05$; ecc $5^{\circ}, p=3.66 \times 10^{-8}$; ecc $10^{\circ}, p=1.24 \times 10^{-6} ;$ ecc $15^{\circ}, p=2.21 \times 10^{-22}$ ).

\section{Discussion}

Previous studies independently suggested a role for either the LGN or pulvinar in blindsight. However, the approaches used in these studies included issues on the variability of the subjects' conditions, such as lesion size, time from lesioning, and how visual capacity was tested. In the present study, we investigated the roles of the pulvinar and LGN in the same monkeys with unilateral V1 lesions. We extensively lesioned the V1 including the white matter and a part of the neighboring V2 by aspiration (Yoshida et al., 2008) to mimic actual blindsight patients
(Weiskrantz et al., 1974; Barbur et al., 1993). Such extensive lesioning of V1 resulted in massive retrograde degeneration of the LGN, but $18-34 \%$ of koniocellular neurons survived 3-8 years after V1 lesioning (Kinoshita et al., 2019). In the present study, it was found that the survival rates of koniocellular neurons in monkey $\mathrm{O}$ (killed at 6 months after V1 lesioning) and monkey $\mathrm{T}$ (killed at 50 months after V1 lesioning) were $52 \%$ and $35 \%$, respectively. In both monkeys, a majority of both magnocellular and parvocellular LGN neurons were already degenerated when the experiments were terminated, whereas a small number of these neuron types appear to have survived as detected by anti-NeuN immunostaining (Fig. 12C,D). We found that the pulvinar and LGN contributed simultaneously to the performance of VGS. If one of the two regions was inactivated by muscimol, the performance of the VGS task was impaired. Their errors in saccades occurred both in the direction and amplitude domains. Conversely, on the intact side, inactivation of the pulvinar did not affect VGS performance, while that of the LGN impaired it. The effective sites on the ipsilesional pulvinar covered a broad area of the pulvinar, and mostly overlapped with those on the contralesional side, which were not effective (Fig. 2C). These results suggested that some plastic changes had occurred in the neural circuits including the pulvinar after V1 lesioning. With the present results, it is still unclear when the pulvinar becomes critical for saccade control after V1 lesioning in relation to the retrograde degeneration of the LGN. To understand that, we need to examine the effects of pulvinar inactivation and histology of the LGN in animals with an even shorter survival time after V1 lesioning than monkey $\mathrm{O}$ (i.e., at $<6$ months after lesioning).

\section{Technical considerations}

Before arguing on the roles of the ipsilesional pulvinar and LGN in blindsight, we need to discuss the possibility that the effect of LGN inactivation was caused by the spread of muscimol to the pulvinar and vice versa. First, as shown in Figure 2, $A$ and $B$, the centers of the injection sites in the pulvinar and LGN were confirmed to be in the core of both nuclei. To assess the possibility of muscimol spread between the LGN and pulvinar, we varied the injection sites among the daily experimental sessions (pulvinar: within an $\sim 4$ $\mathrm{mm}$ range along the mediolateral axis, LGN: within an $\sim 3 \mathrm{~mm}$ range along the anteroposterior axis). In the case of ipsilesional pulvinar inactivation, the effect of inactivation could be observed clearly at its most medial part after 20 min from injection in both monkeys, which was the most remote track from the LGN. Conversely, regarding LGN inactivation, the effect of 
inactivation was observed at the most anterior part of the LGN in monkey $\mathrm{T}$, that is, the most remote part from the pulvinar. In contrast, in monkey $\mathrm{O}$, the effect of inactivation was observed only in the most posterior part of the LGN (the closest to the pulvinar). Furthermore, it took $>60$ min for the effect of LGN inactivation to emerge, which was later than for ipsilesional pulvinar inactivation (twosample $t$ test, $p<0.05)$. These results suggest the possibility that muscimol injected into the LGN could have spread to the pulvinar in monkey $\mathrm{O}$. However, we considered that it is rather unlikely that the monkey shortly after lesioning (monkey O, 1-5 months after lesioning and the LGN was more intact) depended more on the pulvinar than the monkey with a longer survival time after lesioning (monkey $\mathrm{T}, 40-47$ months after lesioning and the LGN was more extensively degenerated). Thus, it is reasonable to consider that the slow action of LGN inactivation was not caused by the spread of muscimol to the pulvinar, but probably because the pulvinar partly compensated for the function of the LGN and a large portion of the LGN needed to be blocked to show behavioral effects.

\section{Roles of the pulvinar and LGN in blindsight}

A recent review (Tamietto and Morrone, 2016) suggested that blindsight is not a single phenomenon, but should be considered as a constellation of functions of various visual pathways that survive V1 lesioning. In our current study, we showed that the pulvinar and LGN play a critical role in VGS task performance. A recent study by Yu et al. (2018) showed clear visual responses in the LGNs of marmosets after V1 lesion, suggesting that not only the koniocellular neurons but also parvocellular and magnocellular LGN neurons survived and were visually responsive 17-33 months after the extensive V1 lesion. In our study, we could also find a small number of surviving neurons in the lesion-affected area of LGN, which was consistent with findings of Yu et al. (2018) on marmosets and Matthews et al. (1960) on macaques. Thus, these surviving magnocellular, parvocellular, and koniocellular neurons could mediate the residual vision after V1 lesion. At this moment, it is still unclear whether LGN and pulvinar are equally critical or whether LGN plays the major role and pulvinar plays just a subsidiary role or vice versa. Another possibility is that the two nuclei play differential roles depending on the visual input property. Many studies have suggested various abilities of subjects with blindsight. For example, blindsight subjects can discriminate the movement of visual stimuli, colors, size, and contrast (Stoerig, 1987; Brent et al., 1994; Cowey and Stoerig, 1995; Weiskrantz, 1995; Benson et al., 1998; Yoshida and Isa, 2015). Furthermore, they can perform more complex tasks such as the short-term memory task and classical conditioning task triggered by visual cues presented in their lesion-affected visual field (Takaura et al., 2011; Takakuwa et al., 2017). All of these studies used a simple dot spot as a cue stimulus. Conversely, several previous studies examined the functions of the LGN with moving visual stimuli (Schmid et al., 2010; Ajina et al., 2015; Ajina and Bridge, 2018). To dissociate the functions of the two regions, experiments are needed in which the features of the visual stimuli, such as luminance contrast and spatial and temporal frequencies, are systematically changed, and the behavioral probes for functional assessment should also be varied.

Regarding the significant reduction in the saccade peak velocity in terms of the main sequence relationship in monkey $\mathrm{O}$, it is possible that differences in brightness between the intact visual field and lesion-affected visual field have an effect on saccade peak velocity. Different brightness conditions have been reported to affect saccade peak velocity in humans (Felssberg and Dombrowe, 2018). In the lesion-affected visual field, the sensitivity of the monkeys to the contrast of the ST was clearly reduced (Fig. 1C). This suggests that low sensitivity to contrast affected saccade velocity, similar to the effect of a dimmed condition. In any case, however, the difference here was small, and the overall results suggested that the effect of inactivation of the ipsilesional pulvinar and LGN on the saccade motor system was the minimum and the effects of inactivation were in the visual or visuomotor transformation process of the VGS control system.

\section{Plasticity in blindsight subjects}

The present study showed that inactivation of the ipsilesional pulvinar, on the same side as the lesioned V1, impaired VGS performance, while that of the contralesional pulvinar, on the side as an intact V1, had no effect. These results suggested that some plastic changes occurred in the visual pathway for VGS to upregulate the contribution of the pulvinar to compensate for the loss of $\mathrm{V} 1$ during recovery. The mechanism for the upregulation of pulvinar function is still unclear. A possible mechanism might be the disinhibition of pulvinar neurons leading to the generation of firing responses to visual stimuli, which was the subthreshold for the induction of spiking responses in pulvinar neurons before V1 lesioning. Conversely, an anatomic study showed that the subregion of the pulvinar receiving inputs from the SC has little overlap with the subregion projecting to the extrastriate cortex such as area MT (Stepniewska et al., 1999). If so, it would be more likely that some plastic changes in axonal trajectories including sprouting occurred in the SC-pulvinar-MT pathway after V1 lesioning. Previous studies showed that the function of the SC on the ipsilesional side is upregulated after V1 lesioning in terms of VGS, memory-guided saccades, and pavlovian conditioning, compared with the contralesional SC (Takaura et al., 2011; Takakuwa et al., 2017, 2018). Considering these results, the plastic changes in transmission through the SC-pulvinar-cortical pathways might underlie blindsight. 

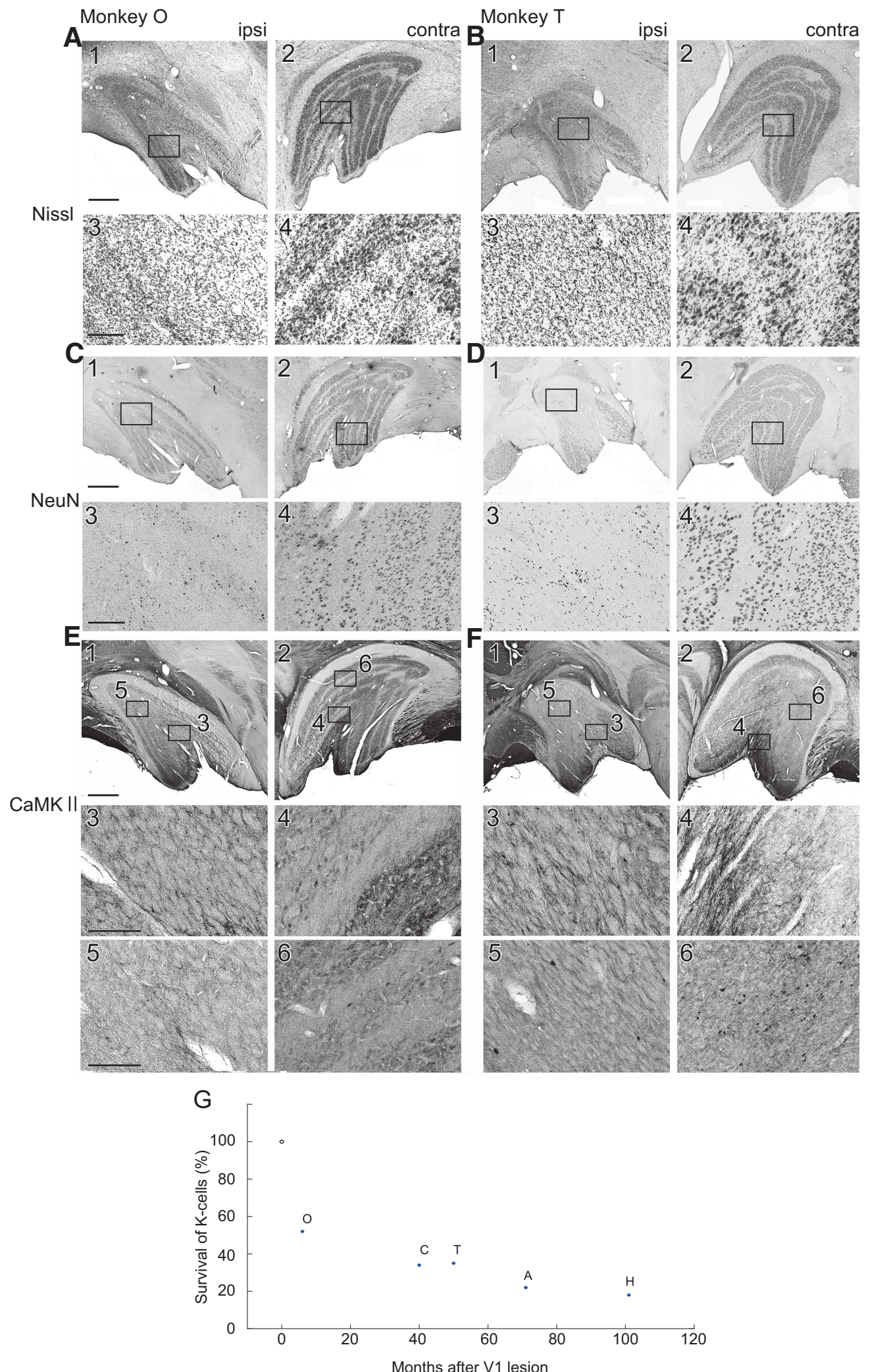

Figure 12. Retrograde degeneration of $L G N$ neurons. $A-F$, LGN neurons on the ipsilesional and contralesional sides in monkey $0(A, C, E)$ and monkey $T(B, D, F)$ are exhibited. $A \mathbf{A}-4$, Low $(\mathbf{1}, \mathbf{2})$

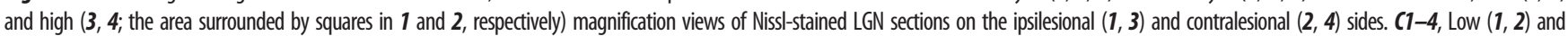
high $(3,4$; the areas surrounded by squares in $\mathbf{1}$ and 2 , respectively) magnification view of sections with anti-NeuN immunostained sections of the LGN on the ipsilesional $(\mathbf{1}, 3)$ and contralesional $(2,4)$ sides. E1-6, Low $(\mathbf{1}, \mathbf{2})$ and high $(3,4,5,6)$ magnification view of sections with anti-CaMKIl $\alpha$ immunostained sections of the LGN on the ipsilesional $(\mathbf{1}, 3,5)$ and contralesional $(2,4,6)$ sides. Panels 3 and 4 are taken from the presumed magnocellular subdivision of koniocellular neurons $(K 1 / K 2)$, and panels 5 and $\mathbf{6}$ are taken from the presumed parvocellular domain of koniocellular neurons (K3-K6). $\boldsymbol{B}, \boldsymbol{D}, \boldsymbol{F}$, The same arrangement as in $\boldsymbol{A}, \boldsymbol{C}$, and $\boldsymbol{E}$ for monkey $T$, respectively. Scale bars: $\boldsymbol{A}-\boldsymbol{F} \mathbf{2}, 1 \mathrm{~mm} ; \boldsymbol{A}-\boldsymbol{D}, \mathbf{3}, \mathbf{4}, 200 \mu \mathrm{m} ; \boldsymbol{E}, \boldsymbol{F}, \mathbf{3 - 6}, 200 \mu \mathrm{m}$, respectively. $\boldsymbol{G}$, Relationship between the survival time of the animal (horizontal axis) and survival rate of koniocellular neurons (vertical axis, "K-cells") for monkeys $0, T, C, A$, and $H$. The data for monkeys $C$, A, and $H$ were taken from our previous report (Kinoshita et al., 2019). A black dot plotted at $(0,100)$ indicates an example of control before lesioning V1. 


\section{References}

Ajina S, Bridge H (2018) Blindsight relies on a functional connection between $\mathrm{hMT}+$ and the lateral geniculate nucleus, not the pulvinar. PLoS Biol 16: e2005769.

Ajina S, Pestilli F, Rokem A, Kennard C, Bridge H (2015) Human blindsight is mediated by an intact geniculo-extrastriate pathway. Elife 4:e08935.

Alkhunizi MS, Fakhoury M, Abou-Kheir W, Lawand N (2020) Gadolinium Retention in the Central and Peripheral Nervous System: Implications for Pain, Cognition, and Neurogenesis, Radiology 297:407-416.

Atapour N, Worthy KH, Lui LL, Yu HH, Rosa MGP (2017) Neuronal degeneration in the dorsal lateral geniculate nucleus following lesions of primary visual cortex: comparison of young adult and geriatric marmoset monkeys. Brain Struct Funct 222:3283-3293.

Barbur JL, Watson JDG, Frackowiak RSJ, Zeki S (1993) Conscious visual perception without VI. Brain 116:1293-1302.

Bender DB (1981) Retinotopic organization of macaque pulvinar. J Neurophysiol 46:672-693.

Bender DB (1983) Visual activation of neurons in the primate pulvinar depends on cortex but not colliculus. Brain Res 279:258-261.

Bender DB (1988) Electrophysiological and behavioral experiments on the primate pulvinar. Prog Brain Res 75:55-65.

Benson PJ, Guo K, Blakemore C (1998) Direction discrimination of moving gratings and plaids and coherence in dot displays without primary visual cortex (V1). Eur J Neurosci 10:3767-3772.

Berman RA, Wurtz RH (2010) Functional identification of a pulvinar path from superior colliculus to cortical area MT. J Neurosci 30:6342-6354.

Brent PJ, Kennard C, Ruddock KH (1994) Residual colourvision in a human hemianope: spectral responses and colour discrimi-nation. Proc Biol Sci 256:219-225.

Bridge H, Bell AH, Ainsworth M, Sallet J, Premereur E, Ahmed B, Mitchell AS, Schüffelgen U, Buckley M, Tendler BC, Miller KL, Mars RB, Parker AJ, Krug K (2019) Preserved extrastriate visual network in a monkey with substantial, naturally occurring damage to primary visual cortex. Elife 8:e42325.

Cowey A, Stoerig P (1989) Projection patterns of surviving neurons in the dorsal lateral geniculate nucleus following discrete lesions of striate cortex: implications for residual vision. Exp Brain Res 75:631-638.

Cowey A, Stoerig P (1995) Blindsight in monkeys. Nature 373:247-249.

Diamond IT, Hall WC (1969) Volution of neocortex. Science 164:251-262.

Felssberg A-M, Dombrowe I (2018) The effect of different brightness conditions on visually and memory guided saccades. Vision Res 142:20-26.

Gattass R, Oswaldo Cruz E, Sousa APB (1978) Visuotopic organization of the Cebus pulvinar: a double representation of the contralateral hemifield. Brain Res 152:1-16.

Gattass R, Galkin TW, Desimone R, Ungerleider LG (2014) Subcortical connections of area V4 in the macaque. J Comp Neurol 522:1941-1965.

Georgy L, Celeghin A, Marzi CA, Tamietto M, Ptito A (2016) The superior colliculus is sensitive to gestalt-like stimulus configuration in hemispherectomy patients. Cortex 81:151-161.

Hendry SH, Yoshioka TA (1994) Neurochemically distinct third channel in the macaque dorsal lateral geniculate nucleus. Science 264:575-577.

Kaas JH, Lyon DC (2007) Pulvinar contributions to the dorsal and ventral streams of visual processing in primates. Brain Res Rev 55:285-296.

Kato R, Takaura K, Ikeda T, Yoshida M, Isa T (2011) Contribution of the retino-tectal pathway to visually guided saccades after lesion of the primary visual cortex in monkeys. Eur J Neurosci 33:1952-1960.

Kinoshita M, Matsui R, Kato S, Hasegawa T, Kasahara H, Isa K, Watakabe A, Yamamori T, Nishimura Y, Alstermark B, Watanabe D, Kobayashi K, Isa $\mathrm{T}$ (2012) Genetic dissection of the circuit for hand dexterity in primates. Nature 487:235-238.

Kinoshita M, Kato R, Isa K, Kobayashi K, Kobayashi K, Onoe H, Isa T (2019) Dissecting the circuit for blindsight to reveal the critical role of pulvinar and superior colliculus. Nat Commun 10:135.
Leh SE, Ptito A, Schönwiesner M, Chakravarty MM, Mullen KT (2010) Blindsight mediated by an S-cone-independent collicular pathway: an fMRI study in hemispherectomized subjects. J Cogn Neurosci 22:670682.

Li K, Patel J, Purushothaman G, Marion RT, Casagrande VA (2013) Retinotopic maps in the pulvinar of bush baby (Otolemur garnettii). J Comp Neurol 521:3432-3450.

Matthews MR, Cowan WM, Powell TP (1960) Transneuronal cell degeneration in the lateral geniculate nucleus of the macaque monkey. J Anat 94:145-169.

Mohler CW, Wurtz RH (1977) Role of striate cortex and superior colliculus in visual guidance of saccadic eye movements in monkeys. J Neurophysiol 40:74-94.

Pöppel E, Held R, Frost D (1973) Residual visual function after brain wounds involving the central visual pathways in man. Nature 243:295-296.

Rodman HR, Gross CG, Albright TD (1990) Afferent basis of visual response properties in area MT of the macaque. II. Effects of superior colliculus removal. J Neurosci 10:1154-1164.

Sanders MD, Warrington EK, Marshall J, Wieskrantz L (1974) Blindsight": vision in a field defect. Lancet 303:707-708.

Savina O, Guitton D (2018) The primitive retino-tecto-reticular pathway is functional in hemidecorticate patients. Curr Biol 28:R1184-R1186.

Schmid MC, Mrowka SW, Turchi J, Saunders RC, Wilke M, Peters AJ, Ye FQ, Leopold DA (2010) Blindsight depends on the lateral geniculate nucleus. Nature 466:373-377.

Stepniewska I, Qi HX, Kaas JH (1999) Do superior colliculus projection zones in the inferior pulvinar project to MT in primates? Eur J Neurosci 11:469-480.

Stoerig P (1987) Chromaticity and achromaticity. Evidence for a functional differentiation in visual field defects. Brain 110:869-886.

Takakuwa N, Kato R, Redgrave P, Isa T (2017) Emergence of visually-evoked reward expectation signals in dopamine neurons via the superior colliculus in V1 lesioned monkeys. Elife 6:e24459.

Takakuwa N, Redgrave P, Isa T (2018) Cortical visual processing evokes short-latency reward-predicting cue responses in primate midbrain dopamine neurons. Sci Rep 8:14984.

Takaura K, Yoshida M, Isa T (2011) Neural substrate of spatial memory in the superior colliculus after damage to the primary visual cortex. J Neurosci 31:4233-4241

Tamietto M, Morrone MC (2016) Visual plasticity: blindsight bridges anatomy and function in the visual system. Curr Biol 26:R70-R73.

Tomaiuolo F, Ptito M, Marzi CA, Paus T, Ptito A (1997) Blindsight in hemispherectomized patients as revealed by spatial summation across the vertical meridian. Brain 120:795-803.

Warner EC, Kwan CW, Wright D, Johnston AL, Egan FG, Bourne AJ (2015) Preservation of vision by the pulvinar following early-life primary visual cortex lesions. Curr Biol 25:424-434.

Yoshida M, Isa T (2015) Signal detection analysis of blindsight in monkeys. Sci Rep 5:10755.

Yoshida M, Takaura K, Kato R, Ikeda T, Isa T (2008) Striate cortical lesions affect deliberate decision and control of saccade: implication for blindsight. J Neurosci 28:10517-10530.

Yu HH, Atapour N, Chaplin TA, Worthy KH, Rosa MGP (2018) Robust visual responses and normal retinotopy in primate lateral geniculate nucleus following long-term lesions of striate cortex. J Neurosci 38:3955-3970.

Wilke M, Turchi J, Smith K, Mishkin M, Leopold DA (2010) Pulvinar inactivation disrupts selection of movement plans. J Neurosci 30:8650-8659.

Weiskrantz L (1995) The problem of animal consciousness in relation to neuropsychology. Behav Brain Res 71:171-175.

Weiskrantz L, Warrington EK, Sanders MD, Marshall J (1974) Visual capacity in the hemianopic field following a restricted occipital ablation. Brain 97:709-728. 